CONFORMAL GEOMETRY AND DYNAMICS

An Electronic Journal of the American Mathematical Society

Volume 10, Pages 257-284 (October 5, 2006)

S $1088-4173(06) 00150-0$

\title{
MATING A SIEGEL DISK WITH THE JULIA SET OF A REAL QUADRATIC POLYNOMIAL
}

\author{
G. BLE AND R. VALDEZ
}

\begin{abstract}
In this work, we show that it is possible to construct the mating between a quadratic polynomial with a Siegel disk and a real quadratic polynomial possessing a postcritical orbit that is semi-conjugate to a rigid rotation with the same rotation number as the Siegel disk.
\end{abstract}

\section{INTRODUCTION}

The mating of two quadratic polynomials is a topological construction, suggested by Douady and Hubbard, that consists of gluing their filled Julia sets along their boundaries, via an equivalence relation, to get a quadratic rational map where it is possible to observe the dynamics of both polynomials $[\mathrm{DH}]$. Explicitly, we take two monic quadratic polynomials $P_{1}$ and $P_{2}$ whose filled Julia sets $K_{i}=K\left(P_{i}\right)$ are locally-connected, where the filled Julia set $K\left(P_{i}\right)$ is the set of $z \in \mathbb{C}$ for which the orbit under $P_{i}$ is bounded. By Böttcher's Theorem there exists a conformal isomorphism $\Phi_{i}$ between the basin of infinity $\widehat{\mathbb{C}} \backslash K_{i}$ and $\widehat{\mathbb{C}} \backslash \overline{\mathbb{D}}$, with $\Phi_{i}(\infty)=\infty$ and $\Phi_{i}^{\prime}(\infty)=1$, such that $\Phi_{i}$ conjugates $P_{i}$ to the map $z \mapsto z^{2}$; that is, the following diagram commutes:

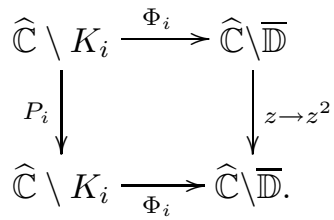

By Carathéodory's Theorem the inverse map $\Phi_{i}^{-1}$ has a continuous extension to the closure of $\widehat{\mathbb{C}} \backslash \overline{\mathbb{D}}$. This extension induces a parametrization of the Julia set $J_{i}=\partial K_{i}$, defined by

$$
\Gamma_{i}(t)=\Phi_{i}^{-1}\left(e^{2 \pi i t}\right): \mathbb{T}=\mathbb{R} / \mathbb{Z} \rightarrow J_{i},
$$

and it is commonly referred to as the Carathéodory loop of $J_{i}$. By definition, $\Gamma_{i}(2 t)=P_{i}\left(\Gamma_{i}(t)\right)$ for all $t \in \mathbb{T}$ and $i=1,2$.

Let $X$ be the topological space obtained by the disjoint union of $K_{1}$ and $K_{2}$, gluing the two filled Julia sets along their Carathéodory loops in reverse directions,

Received by the editors February 10, 2006.

2000 Mathematics Subject Classification. Primary 37F10; Secondary 37F45, 37F50.

Key words and phrases. Holomorphic dynamics, rational map, mating, Julia set, Mandelbrot set.

The first author was supported by CONACYT, 42249 .

The second author was supported by PROMEP, UAEMOR-PTC-166.

(C)2006 American Mathematical Society Reverts to public domain 28 years from publication 
i.e.,

$$
X=\left(K_{1} \sqcup K_{2}\right) /\left(\Gamma_{1}(t) \sim \Gamma_{2}(-t)\right) .
$$

If $X$ is homeomorphic to the 2-sphere $\mathbf{S}^{2}$, then the pair of polynomials $\left(P_{1}, P_{2}\right)$ is called topologically mateable and the induced map of $\mathbf{S}^{2}$,

$$
P_{1} \perp_{\tau} P_{2}=\left(\left.\left.P_{1}\right|_{K_{1}} \sqcup P_{2}\right|_{K_{2}}\right) /\left(\Gamma_{1}(t) \sim \Gamma_{2}(-t)\right)
$$

is the topological mating of $P_{1}$ and $P_{2}$. In particular, there are canonical semiconjugacies $K_{1} \rightarrow K_{1} \perp_{\tau} K_{2}$ and $K_{2} \rightarrow K_{1} \perp_{\tau} K_{2}$, from $P_{1}$ and $P_{2}$ to $P_{1} \perp_{\tau} P_{2}$, respectively.

In general, $X$ is not homeomorphic to $\mathbf{S}^{2}$ TL. However, we will see below that in many cases $X$ is a topological sphere and $P_{1} \perp_{\tau} P_{2}$ is a degree 2 branched covering of the sphere. Then, it will be natural to ask whether it possesses an invariant conformal structure.

Definition 1.1. A quadratic rational map $R: \widehat{\mathbb{C}} \rightarrow \widehat{\mathbb{C}}$ is called a mating of $P_{1}$ and $P_{2}$,

$$
R=P_{1} \perp P_{2},
$$

if it is conjugate to the topological mating $P_{1} \perp_{\tau} P_{2}$ by an homeomorphism which is conformal in the interiors of $K_{1}$ and $K_{2}$. If such $R$ is unique up to conjugation by a Möbius transformation, then we refer to it as the mating of $\left(P_{1}, P_{2}\right)$.

We mention that there are other equivalent methods to formulate the mating M1, YaZa].

1.1. Examples of matings. Let $c_{i} \in \mathbb{C}$ and $P_{i}(z)=z^{2}+c_{i}$. The Mandelbrot set $M$ is the set of $c_{i} \in \mathbb{C}$ for which $K_{i}=K\left(P_{i}\right)$ is connected. Let $W_{0}$ be the main hyperbolic component of $M$ and let $\partial W_{0}$ (the main cardioid) be the boundary of $W_{0}$. It is known that there exists a conformal isomorphism between $W_{0}$ and $\mathbb{D}$, this isomorphism can be extended continuously to $\partial W_{0}$ and this defines the internal argument $\gamma$ for all $c$ in the main cardioid [D. If $c \in \partial W_{0}$ has a rational internal argument $\frac{p}{q},(p, q)=1$, then $c$ is a root of a hyperbolic component of period $q$. In this case, $M \backslash\{c\}$ has two components, the one that does not contain $W_{0}$ is called the $\frac{p}{q}$-limb and we denote it by $W_{\frac{p}{q}}$. If $\frac{p_{1}}{q_{1}}$ and $\frac{p_{2}}{q_{2}}$ are two positive rational numbers such that $\frac{p_{1}}{q_{1}}+\frac{p_{2}}{q_{2}}=1$, then we call $W_{\frac{p_{1}}{q_{1}}}, W_{\frac{p_{2}}{q_{2}}}$ a pair of conjugate limbs of the Mandelbrot set.

Theorem 1.1 (Lei, Rees, and Shishikura [TL, Re, Sh]). Let $c_{1}, c_{2}$ be two parameter values in $M$ not in conjugate limbs of the Mandelbrot set such that $P_{c_{1}}$ and $P_{c_{2}}$ have postcritically finite sets. Then $P_{c_{1}}$ and $P_{c_{2}}$ are topologically mateable. Moreover, their mating $F=P_{c_{1}} \perp P_{c_{2}}$ exists.

If the critical point is pre-periodic, the Julia set is a dendrite with no interior and the mating of two dendrites is a Lattès map. An example of this case was studied by Milnor in [M1].

Using quasiconformal surgery, Theorem 1.1 can be extended to any pair $P_{c_{1}}$, $P_{c_{2}}$, where the $c_{i}$ belong to hyperbolic components $W_{1}, W_{2}$ of the Mandelbrot set which do not belong to conjugate limbs. This procedure gives an isomorphism between the product $W_{1} \times W_{2}$ and a hyperbolic component in the parameter space of quadratic rational maps. This isomorphism, however, does not necessarily extend continuously to the product of closures $\overline{W_{1}} \times \overline{W_{2}}, \mathrm{Ep}$. 
One of the first examples of mating for parameters in the boundary of the Mandelbrot set has been given by Yampolsky and Zakeri in YYaZ. They consider the mating between quadratic polynomials with a Siegel disk in their filled Julia sets. For this, we consider an irrational number $0<\theta<1$ and let $\left[a_{1}, a_{2}, a_{3}, \ldots\right]$ be its continued fraction; that is,

$$
\theta=\frac{1}{a_{1}+\frac{1}{a_{2}+\frac{1}{a_{3}+\ldots}}}
$$

We say that $\theta$ is of bounded type if the sequence $\left\{a_{n}\right\}$ is bounded. Let $f_{\theta}(z)=$ $e^{2 \pi i \theta} z+z^{2}$.

Theorem 1.2 (Yampolsky and Zakeri YaZa). Let $0<\theta, \nu<1$ be two irrational numbers of bounded type. If $\theta \neq 1-\nu$, then the polynomials $f_{\theta}$ and $f_{\nu}$ are topologically mateable. Moreover, there exists a quadratic rational map $F$ such that $F=f_{\theta} \perp f_{\nu}$.

1.2. Parameters for the mating. Since the Mandelbrot set is locally-connected at every point $c \in \partial W_{0}$, we have an external ray of the Mandelbrot set $R_{M}(\theta)$ which lands at $c$, for all $c \in \partial W_{0}$. We define the following set:

$$
\operatorname{Arg}\left(W_{0}\right)=\left\{\theta \in[0,1 / 3]: R_{M}(\theta) \text { lands at } c \in \partial W_{0}\right\}
$$

Let $c$ be a parameter in the main cardioid with irrational internal argument $\gamma$ and external argument $\theta \in[0,1 / 3]$. If $P_{c}$ is linearizable near the $\alpha_{c}$ fixed point, it has a Siegel disk $\Delta$. When $J_{c}$ is locally connected, we have that $0 \in \partial \Delta$ and the dynamical external ray of argument $\theta$ lands at $c \in \partial \Delta[\mathrm{D} 2$.

Notice that the orbit of $\theta$ under the angle doubling map does not meet the interval $\left[\frac{1}{4}+\frac{\theta}{4}, \frac{1}{2}+\frac{\theta}{4}\right]$ since the boundary of $\Delta$ is invariant under $P_{c}$, the angles $\frac{1}{4}+\frac{\theta}{4}$ and $\frac{1}{2}+\frac{\theta}{4}$ are the external arguments of the first pre-image in $\partial \Delta$ of the critical point and $\left.P_{c}\right|_{J_{c}}$ is semi-conjugate to $t \mapsto 2 t$. In particular, the orbit of $\theta$ does not meet the interval $\left[\frac{1}{2}-\frac{\theta}{4}, \frac{1}{2}+\frac{\theta}{4}\right]$. Since this property is satisfied by all the external rays landing at the critical value of any real parameter $c^{\prime}$ on the boundary of $M$, Douady conjectured that if $\theta$ belongs to $\operatorname{Arg}\left(W_{0}\right)$ and

$$
T(\theta)=\frac{1}{2}+\frac{\theta}{4}
$$

then $T(\theta)$ is an external argument of a real parameter in the Mandelbrot set.

In [B] , it is shown that the external ray with angle $T(\theta)$ lands at $\partial M \cap \mathbb{R}$ for all $\theta \in \operatorname{Arg}\left(W_{0}\right)$. Moreover, if $c$ is in the main cardioid with irrational internal argument $\gamma \in \mathbb{T}$ and external argument $\theta \in \operatorname{Arg}\left(W_{0}\right)$, the following theorem is proved.

Theorem 1.3 (Ble [B] $)$. Let $0<\gamma<1$ be an irrational number. Then the external ray $R_{M}(T(\theta))$ lands at the real parameter $c^{\prime}$; and,

(1) $\left.P_{c^{\prime}}\right|_{\mathcal{O}_{c^{\prime}}(0)}$ is semi-conjugate to a rigid rotation of angle $\gamma$ and

(2) the Mandelbrot set is locally connected at $c^{\prime}$, where $\mathcal{O}_{c^{\prime}}(0)$ denotes the postcritical orbit of $P_{c^{\prime}}(z)=z^{2}+c^{\prime}$.

In this work, we prove the following result. 
Main Theorem. Let $\gamma$ be an irrational number and let $c \in \partial W_{0}$ be a parameter with internal argument $\gamma$. If $\gamma$ is of bounded type, then the mating between $P_{c}$ and $P_{c^{\prime}}$ exists, where $c^{\prime}$ is the parameter given by Theorem 1.3 .

When $\gamma$ is an irrational number, the real quadratic polynomial $P_{c^{\prime}}$, obtained via the transformation $T$, has the property that $K_{c^{\prime}}=J_{c^{\prime}}$ and the critical point is strongly recurrent. Thus, the Main Theorem gives a family of examples of mating between quadratic polynomials with Siegel disks and quadratic polynomials with strongly recurrent critical point. Using the following theorem of Petersen-Zakeri, the Main Theorem can be extended to a set of irrational numbers with full Lebesgue measure in $\left[0, \frac{1}{2}\right]$.

Theorem 1.4 ([PeZa $)$. Let $\gamma=\left[a_{1}, a_{2}, \ldots\right]$ be an irrational number which satisfies the arithmetical condition

$$
\log a_{n}=\mathcal{O}(\sqrt{n}) \text { as } n \rightarrow \infty .
$$

Then, $J\left(P_{c}\right)$ is locally connected and it has Lebesgue measure zero.

In the proof of the Main Theorem, we will use the condition of bounded type in order to have a quasisymmetric conjugation in $\mathbb{T}$ which can be quasiconformally extended to the unit disk. In order to prove Theorem 1.4, Petersen and Zakeri use the model in $\mathrm{Pe}$ and construct the quasiconformal conjugation in the unit disk using David's Theorem, neglecting the condition of bounded type. Hence, the proof of the Main Theorem implies the following.

Theorem. Let $\gamma=\left[a_{1}, a_{2}, \ldots\right]$ be an irrational number. Suppose that $\gamma$ satisfies the arithmetical condition

$$
\log a_{n}=\mathcal{O}(\sqrt{n}) \text { as } n \rightarrow \infty .
$$

Then, the mating between $P_{c}$ and $P_{c^{\prime}}$ exists, where $c \in \partial W_{0}$ has internal argument $\gamma$ and $c^{\prime}$ is the parameter given by Theorem 1.3 .

\section{BACKGROUND MATERIAL}

\subsection{Notations and terminology.}

- The unit disk in the complex plane will be denoted by $\mathbb{D}$ and its boundary, the unit circle, by $\mathbb{T}$. The upper-half plane will be denoted by $\mathbb{H}$.

- For a set $X$ in the plane, we denote by $\bar{X}, \operatorname{int}(X)=\stackrel{\circ}{X}$ and $\partial X$, the closure, the interior and the boundary of $X$, respectively.

- We will denote by $D_{r}(p)$ the disk of radius $r$ with center $p$.

- We denote by $[a, b]$ the closed interval with end-points $a$ and $b$ in $\mathbb{R}$, without specifying their order.

- For two points $a, b$ on the circle which are not diagonally opposite, $[a, b]$ will denote the shorter of the two closed arcs connecting them.

- For $K>1$, we say that two real numbers $a$ and $b$ are $K$-commensurable if

$$
K^{-1} \leq|a| /|b| \leq K .
$$

- Let $R_{t}: \mathbb{T} \rightarrow \mathbb{T}$ be the rigid rotation $x \mapsto x+t(\bmod \mathbb{Z})$.

- We will denote the iteration of a function $f$ with itself $n$ times by

$$
f^{n}=f^{\circ n}=\underbrace{f \circ \cdots \circ f}_{n} .
$$


- Let $\mathcal{O}_{f}\left(z_{0}\right)$ denote the orbit of the point $z_{0}$ under $f$; that is,

$$
\mathcal{O}_{f}\left(z_{0}\right)=\left\{z_{0}, f\left(z_{0}\right)=z_{1}, f\left(z_{1}\right)=z_{2}, \ldots\right\} .
$$

- We set $P_{c}(z)=z^{2}+c$.

- Let $K_{c}=K\left(P_{c}\right)=\left\{z \in \mathbb{C}: \mathcal{O}_{P_{c}}(z)\right.$ is bounded $\}$ denote the filled Julia set of $P_{c}$.

- Let $J_{c}=J\left(P_{c}\right)=\partial K_{c}$ denote the Julia set of $P_{c}$.

2.2. Critical circle map. In this paper we identify the affine manifold $\mathbb{T}=\mathbb{R} / \mathbb{Z}$ with the unit circle

$$
\mathbf{S}^{1}=\{z \in \mathbb{C}:|z|=1\},
$$

using the canonical projection from the real line given by $x \rightarrow e^{2 \pi i x}$.

Definition 2.1. A critical circle map is an orientation-preserving homeomorphism $f: \mathbb{T} \rightarrow \mathbb{T}$ of class $\mathcal{C}^{3}$ with at least one critical point.

A family of examples of these maps is provided by the restriction to $\mathbb{T}$ of the family of degree 3 Blaschke products

$$
Q_{t}(z)=e^{2 \pi i t} z^{2}\left(\frac{z-3}{1-3 z}\right) .
$$

These maps have a single critical point at $1 \in \mathbb{T}$. However, we will consider maps with two critical points in $\mathbb{T}$ for our mating model.

Let $f$ be an orientation-preserving homeomorphism of the circle and let $\hat{f}: \mathbb{R} \rightarrow$ $\mathbb{R}$ be a lift of $f$ with critical points at integer translates of $\hat{c}$, where $c$ is a critical point of $f$. The quantity

$$
\rho(f)=\lim _{n \rightarrow \infty} \frac{\hat{f}^{\circ n}(x)}{n}(\bmod 1),
$$

is independent both of the choice of $x \in \mathbb{R}$ and the lift $\hat{f}$ of a critical circle map $f$, and is referred to as the rotation number of $f$. Moreover, the rotation number is rational of the form $\rho(f)=p / q$ if and only if $f$ has an orbit of period $q$ dMvS].

Proposition 2.1. Let $f_{\lambda}$ be a continuous family of critical circle maps. Then, the map $\lambda \mapsto \rho\left(f_{\lambda}\right)$ is continuous.

To illustrate the connection between the number-theoretic properties of $\rho(f)$ and the dynamics of $f$, let us introduce the notion of a closest return of the critical point $c$. The iterate $f^{\circ n}(c)$ is a closest return, or equivalently, $n$ is a closest return moment, if the interior of the $\operatorname{arc}\left[f^{\circ n}(c), c\right]$ contains no iterates $f^{\circ j}(c)$ with $j<n$. Consider the representation of $\rho(f)$ as a (possibly finite) continued fraction

$$
\rho(f)=\frac{1}{a_{1}+\frac{1}{a_{2}+\frac{1}{a_{3}+\ldots}},}
$$

where the numbers $a_{i}$ are positive integers, and we write as before

$$
\rho(f)=\left[a_{1}, a_{2}, a_{3}, \ldots\right] .
$$

The $n$th convergent of the continued fraction of $\rho(f)$ is the rational number

$$
\frac{p_{n}}{q_{n}}=\left[a_{1}, a_{2}, \ldots, a_{n}\right]
$$


written in reduced form. We set $p_{0}=0, q_{0}=1$. One can easily see the recursive relations

$$
\begin{aligned}
p_{n} & =a_{n} p_{n-1}+p_{n-2}, \\
q_{n} & =a_{n} q_{n-1}+q_{n-2},
\end{aligned}
$$

for $n \geq 2\left[\mathrm{HW}\right.$. In this notation, the iterates $\left\{f^{\circ q_{n}}(c)\right\}$ are the consecutive closest returns of the critical point $c$.

Theorem 2.1 (Yoccoz $[\mathrm{Y}]$ ). Let $f$ be an analytic critical circle map with irrational rotation number $\rho(f)=t$. Then there exists a homeomorphism $h: \mathbb{T} \rightarrow \mathbb{T}$ such that $h \circ f=R_{t} \circ h$.

Definition 2.2. A homeomorphism $g: \mathbb{R} \rightarrow \mathbb{R}$ is called $K$-quasisymmetric if

$$
0<K^{-1} \leq \frac{|h(x+t)-h(x)|}{|h(x)-h(x-t)|} \leq K<+\infty
$$

for all $x \in \mathbb{R}$ and all $t>0$.

A homeomorphism $f: \mathbb{T} \rightarrow \mathbb{T}$ is $K$-quasisymmetric if its lift $\hat{f}$ is $K$-quasisymmetric. In the following result of Herman and Świastek, we suppose that $f$ has a finite number of critical points and we further assume that all the critical points are of cubic type.

Theorem 2.2 (Herman-Świątek [Pe1]). A critical circle map $f$ is conjugate to a rigid rotation by a quasisymmetric homeomorphism $h$ if and only if the rotation number $\rho(f)$ is irrational of bounded type.

The proof for this result when $f$ has a unique critical point has been given by Petersen in Pe1, but the proof also holds for critical circle maps with a finite number of critical points.

The above result is based on a set of estimates on the small-scale geometry of critical circle maps. These Herman-Światek real a priori bounds became a key element of renormalization and rigidity results for critical circle maps, and will play an important role in the proof of the Main Theorem.

For a critical circle map with an irrational rotation number and a critical point $c$, we denote by $I_{n}$ the $n$th closest return interval $\left[c, f^{\circ q_{n}}(c)\right]$.

Remark 2.1. For every $n>1$, the closed intervals

$$
I_{n-1}, f\left(I_{n-1}\right), \ldots, f^{\circ q_{n}-1}\left(I_{n-1}\right), I_{n}, f\left(I_{n}\right), \ldots, f^{\circ q_{n-1}-1}\left(I_{n}\right)
$$

cover the entire circle and have disjoint interiors.

From this, we obtain a partition of $\mathbb{T}$, which is called the dynamical partition of level $n$ associated to $f$. This partition can be done with respect to any critical point $c$ of $f$.

Remark 2.2. The consecutive closest returns $f^{\circ q_{n}}(c)$ and $f^{\circ q_{n+1}}(c)$ occur on different sides of the critical point $c$; that is, $c \in\left(f^{\circ q_{n}}(c), f^{\circ q_{n+1}}(c)\right)$. 
Herman-Świątek real a priori bounds. There exists $K>1$ such that, for every critical circle map $f$ with an irrational rotation number, the following holds: There exists $N=N(f)>0$ such that, for every $n>N$, the adjacent elements of the dynamical partition of level $n$ are $K$-commensurable. In particular,

$$
K^{-1}\left|I_{n}\right| \leq\left|I_{n+1}\right| \leq K\left|I_{n}\right| .
$$

As a consequence, for every $M>0$ there exists a universal constant $K_{M}>1$ such that the following holds: For all sufficiently large $n$, the arcs

$$
\left[f^{\circ q_{n-1}+(j-1) q_{n}}(c), f^{\circ q_{n-1}+j q_{n}}(c)\right], \quad\left[f^{-(j-1) q_{n}}(c), f^{-j q_{n}}(c)\right] \quad \text { and } \quad\left[c, f^{\circ q_{n-1}}(c)\right]
$$

are $K_{M}$-commensurable, for $1 \leq j \leq a_{n+1}-1$ with $\min \left(j, a_{n+1}-j\right)<M$, where $\left[a_{1}, a_{2}, \cdots\right]$ is the continued fraction of $\rho(f)$ and $\frac{p_{n}}{q_{n}}$ is the $n$th convergent [Pe1].

2.3. Prime ends. Certain concepts and results about the theory of prime ends are necessary for this work. Let $D$ denote a bounded simply-connected domain in $\mathbb{C}$. A simple Jordan arc with one end-point on $\partial D$ and all its other points in $D$ is called an end-cut of $D$. A cross-cut of $D$ is a Jordan arc that lies in $D$ except for its two end-points or a Jordan curve that lies in $D$ except for one point.

A point in the boundary of $D$ is accessible from $D$ if it is an end-point of an end-cut in $D$.

Definition 2.3. A sequence $q_{1}, q_{2}, \ldots, q_{n}, \ldots$ of cross-cuts of $D$ is called a chain if the following conditions are satisfied:

(1) They are pairwise disjoint, even if they are considered with their end-points.

(2) Any cross-cut $q_{n}$ separates $D$ into two domains, one of which contains $q_{n-1}$ and the other $q_{n+1}$.

(3) The diameter of $q_{n}$ tends to zero as $n$ goes to infinity.

It follows from (2) that $q_{n}$ determines two subdomains of $D$; hence, we will denote by $d_{n}$ the one that contains all the cross-cuts $q_{m}$, with $m>n$.

Definition 2.4. Two chains $C_{1}=\left\{q_{n}\right\}$ and $C_{2}=\left\{q_{n}^{\prime}\right\}$ are equivalent if for all $n \in \mathbb{N}$, the domain $d_{n}$ contains all but a finite number of the cross-cuts $q_{n}^{\prime}$ and the domain $d_{n}^{\prime}$ defined by $q_{n}^{\prime}$ contains all but a finite number of the cross-cuts $q_{n}$.

A prime end of $D$ is an equivalent class of chains in $D$. A chain belonging to such class is said to belong to the prime end, which we shall generally denote by $P$.

Let $P$ be a prime end of $D,\left\{q_{n}\right\}$ a chain belonging to $P, d_{n}$ the subdomain of $D$ defined by $q_{n}$ and containing $q_{n+1}$. If $q_{n}^{\prime}$ is an equivalent chain to $q_{n}$, then

$$
\bigcap \bar{d}_{n}=\bigcap \overline{d_{n}^{\prime}} \text {. }
$$

The set

$$
I(P)=\bigcap \bar{d}_{n}
$$

is called the impression of the prime end $P$.

Note that $I(P)$ is either a continuum or a single point and $I(P)$ is contained in $\partial D[\mathrm{CL}]$.

A point $p \in I(P)$ is a principal point relative to the prime end $P$ of $D$ if every neighborhood $D_{r}(p)$ contains a cross-cut $q$ of $D$ belonging to a chain $\left\{q_{n}\right\}$ belonging to $P$. Since every chain belonging to $P$ has at least one limit point in $I(P)$, the set $\Pi(P)$ of principal points of $I(P)$ is not empty and is closed. 
Let $L=L(t), 0 \leq t \leq 1$, be a continuous curve in $D$ converging to $P$; that is, given any chain $\left\{q_{n}\right\}$ belonging to $P$ and any large $N$, there exists $t_{N}<1$ such that $L(t) \subset d_{N}$ for $t \in\left(t_{N}, 1\right)$, where $\left\{d_{n}\right\}$ is the nested sequence of domains defined by $\left\{q_{n}\right\}$. When $L$ converges to $P$, we write $L \operatorname{cgt} P$.

Definition 2.5. We say that a point $w \in I(P)$ is an accessible point of $\partial D$ relative to $P$, if there is an end-cut $L(t) \operatorname{cgt} P$ with

$$
w=\lim _{t \rightarrow 1} L(t)
$$

as its end-point.

Remark 2.3. If the impression $I(P)$ is a single point $b$, then every curve $L \operatorname{cgt} P$ is an end-cut to the accessible point $b$.

Theorem $2.3([\mathrm{CL}])$. A prime end $P$, whose impression $I(P)$ contains an accessible point relative to $P$, has only one principal point. Moreover, there can be at most one accessible point relative to $P$ which, if it exists, is the only principal point.

2.4. Quadratic rational maps. In this part, we give a summary of some relevant results about the space $\mathcal{R}_{2}$ of quadratic rational maps, which can be consulted for more detail in [M2]. A quadratic rational map $R \in \mathcal{R}$ at 2 has the form

$$
R(z)=\frac{p(z)}{q(z)}=\frac{a_{0} z^{2}+a_{1} z+a_{2}}{b_{0} z^{2}+b_{1} z+b_{2}},
$$

where $a_{0}$ and $b_{0}$ are not zero at the same time and $p(z), q(z)$ are two co-prime polynomials. This gives us a natural identification of the space $\mathcal{R} t_{2}$ with an open set in the Zariski topology of $\mathbb{C P}^{5}$. It consists of all points

$$
\left(a_{0}: a_{1}: a_{2}: b_{0}: b_{1}: b_{2}\right) \in \mathbb{C P}^{5}
$$

for which

$$
\operatorname{res}(p / q)=\operatorname{Det}\left\{\begin{array}{cccc}
a_{0} & a_{1} & a_{2} & 0 \\
0 & a_{0} & a_{1} & a_{2} \\
b_{0} & b_{1} & b_{2} & 0 \\
0 & b_{0} & b_{1} & b_{2}
\end{array}\right\} \neq 0 .
$$

We know that the group of Möbius transformations $\mathcal{R a t}_{1} \cong \mathbf{P S L}(2, \mathbb{C})$ acts on the space $\mathcal{R a t}_{2}$ by conjugation; that is, for $g \in \mathcal{R}$ at ${ }_{1}$ and $R \in \mathcal{R}_{2} t_{2}$, we associate $g \circ R \circ g^{-1} \in \mathcal{R a t}_{2}$.

Definition 2.6. We say that $R_{1} \in \mathcal{R}$ at $_{2}$ is holomorphically conjugate to $R_{2} \in \mathcal{R} a_{2}$ if they are in the same orbit under $\mathcal{R}_{1}$.

We denote by $\mathcal{M}_{2}$ the space of all holomorphic conjugacy classes $\langle R\rangle$.

Remark 2.4. The action of $\operatorname{PSL}(2, \mathbb{C})$ on $\mathcal{R a t}_{2}$ is not free. For instance, the Möbius map $g(z)=-z$ acts trivially on the set of odd quadratic rational maps.

By this remark, we have that the space $\mathcal{M}_{2}$ has singularities. However, this space can be identified with the complex affine space $\mathbb{C}^{2} \mathrm{M} 2$. To describe this affine structure, we consider $R \in \mathcal{R}$ at 2 and its three fixed points $z_{1}, z_{2}, z_{3} \in \widehat{\mathbb{C}}$ (which are not necessarily distinct). We denote by $\rho_{i}$ the multiplier of $R$ at the point $z_{i}$ and by

$$
\sigma_{1}=\rho_{1}+\rho_{2}+\rho_{3}, \quad \sigma_{2}=\rho_{1} \rho_{2}+\rho_{1} \rho_{3}+\rho_{2} \rho_{3}, \quad \sigma_{3}=\rho_{1} \rho_{2} \rho_{3},
$$

the elementary symmetric functions. 
Proposition 2.2. The three multipliers determine $R$ up to a Möbius conjugacy and verify the following relation:

$$
\sigma_{3}=\sigma_{1}-2 .
$$

Thus, the space $\mathcal{M}_{2}$ is canonically isomorphic to $\mathbb{C}^{2}$ with coordinates $\sigma_{1}$ and $\sigma_{2}$.

Remark 2.5. For the parameters $\rho_{1}, \rho_{2}$, and $\rho_{3}$, we have:

(1) If $\rho_{1} \rho_{2} \neq 1$, there exists a unique quadratic rational map $F$, up to a Möbius conjugacy, with three different fixed points and $\rho_{3}=\left(2-\rho_{1}-\rho_{2}\right) /\left(1-\rho_{1} \rho_{2}\right)$.

(2) If $\rho_{1} \rho_{2}=1$, then $z_{1}=z_{2}$ and $\rho_{3}$ is arbitrary.

This implies that two parameters suffice to describe all the equivalence classes of quadratic rational maps up to a Möbius conjugacy.

We consider as a special case, the two-parameter family of quadratic rational maps that have one fixed point at zero with multiplier $\lambda$ and critical points at 1 and -1 . An element of this family can be written as:

$$
R_{\lambda, a}(z)=\frac{\lambda z}{z^{2}+a z+1} .
$$

In particular, we are interested in the case when $\lambda=e^{2 \pi i \gamma}$ and $\gamma$ is an irrational number of bounded type.

\section{Petersen's MOdel}

In order to show some properties of the filled Julia sets $K_{c}$ with Siegel disks and bounded rotation numbers, Herman and others have used as a model, the Julia set of the following Blaschke product [D2, $\mathrm{Pe}, \mathrm{Ya}, \mathrm{YaZa}$ :

$$
Q_{t}: z \longmapsto e^{2 \pi i t} z^{2}\left(\frac{z-3}{1-3 z}\right) .
$$

This map has the following properties, proved in $\mathrm{Pe}$ :

(1) $Q_{t}$ leaves $\mathbb{T}$ invariant. The restriction of $Q_{t}$ to $\mathbb{T}$ is a critical circle map with critical point of cubic type at 1 and critical value $e^{2 \pi i t} \in \mathbb{T}$.

(2) For each irrational number $0<\gamma<1$, there exists a unique value $t(\gamma)$ such that the rotation number $\rho\left(\left.Q_{t(\gamma)}\right|_{\mathbb{T}}\right)$ is $\gamma$. Let us set $Q_{\gamma}=Q_{t(\gamma)}$.

(3) $Q_{\gamma}$ has two super-attracting fixed points at 0 and $\infty$.

(4) Let $A_{\gamma}(0)$ and $A_{\gamma}(\infty)$ be the basins of attraction of 0 and $\infty$, respectively. Then there exists a unique isomorphism $\phi$ of $A_{\gamma}(\infty)$ onto $\widehat{\mathbb{C}} \backslash \overline{\mathbb{D}}$ such that $\phi(\infty)=\infty$, tangent to the identity at infinity, which conjugates $Q_{\gamma}$ on $A_{\gamma}(\infty)$ to $z \mapsto z^{2}$ on $\widehat{\mathbb{C}} \backslash \overline{\mathbb{D}}$.

(5) The isomorphism $\phi$ allows us to define the external rays and the equipotentials of the compact and full set $\widehat{\mathbb{C}} \backslash A_{\gamma}(\infty)$.

(6) $A_{\gamma}(\infty)=\widehat{\mathbb{C}} \backslash \overline{\bigcup_{n \geq 0} Q_{\gamma}^{-n}(\mathbb{D})}$.

3.1. Quasi-conformal surgery. By Yoccoz's Theorem 2.1, there exists a unique homeomorphism $h: \mathbb{T} \rightarrow \mathbb{T}$ that conjugates $\left.Q_{\gamma}\right|_{\mathbb{T}}$ to the rigid rotation $R_{\gamma}$ of angle $\gamma$. Moreover, if $\gamma$ is of bounded type and $h(1)=1$, it follows by Herman-Świątek's Theorem 2.2, that $h$ is unique and quasisymmetric. By Douady-Earle's Theorem, 
$h$ can be extended to the unit disk as a quasiconformal map $H_{p}$ (see [DE]). It is possible to modify the Blaschke product $Q_{\gamma}$ to obtain a new map

$$
\tilde{Q}_{\gamma}(z)= \begin{cases}Q_{\gamma}(z), & |z|>1 \\ H_{p}^{-1} \circ R_{\gamma} \circ H_{p}(z), & |z| \leq 1\end{cases}
$$

This map is a quasiregular degree 2 branched covering of $\widehat{\mathbb{C}}$ and it is holomorphic in the complement of the unit disk $\mathbb{D}$. Since the point at infinity is a super-attracting fixed point for $\tilde{Q}_{\gamma}$, we can define the "filled Julia set" of $\tilde{Q}_{\gamma}$ by

$$
K\left(\tilde{Q}_{\gamma}\right)=\left\{z \in \mathbb{C}: \mathcal{O}_{\tilde{Q}_{\gamma}}(z) \text { is bounded }\right\}
$$

and the "Julia set" of $\tilde{Q}_{\gamma}$ as $J\left(\tilde{Q}_{\gamma}\right)=\partial K\left(\tilde{Q}_{\gamma}\right)$. By property (6) of $Q_{\gamma}, K\left(\tilde{Q}_{\gamma}\right)$ is equal to $\widehat{\mathbb{C}} \backslash A_{\gamma}(\infty)$, then $J\left(\tilde{Q}_{\gamma}\right)=\partial A_{\gamma}(\infty)$.

We will perform a quasiconformal surgery that changes the standard conformal structure $\sigma_{0}$ of $\widehat{\mathbb{C}}$ in such a way that the map $\tilde{Q}_{\gamma}$ becomes holomorphic for the new conformal structure. That is, we define a $\tilde{Q}_{\gamma}$-invariant conformal structure $\sigma_{\gamma}$ on the plane as follows. In a first step, we define on $\mathbb{D}$ the structure $\sigma_{\gamma}=H_{p}^{*}\left(\sigma_{0}\right)$, the pullback of the standard structure. For each $n \geq 1$, we define $\sigma_{\gamma}=\left(\tilde{Q}_{\gamma}^{\circ n}\right)^{*}\left(\sigma_{\gamma} \mid \mathbb{D}\right)$ on each component $U_{n}$ of $\left\{\tilde{Q}_{\gamma}^{-n}(\mathbb{D})\right\}$. Since $\tilde{Q}_{\gamma}$ is holomorphic on $\widehat{\mathbb{C}} \backslash \overline{\mathbb{D}}$, this procedure does not increase the dilatation of $\sigma_{\gamma}$. Finally, let $\sigma_{\gamma}=\sigma_{0}$ on the complement of $\bigcup_{n \geq 0} \tilde{Q}_{\gamma}^{-n}(\mathbb{D})$.

Since $R_{\gamma}$ preserves $\sigma_{0}, \tilde{Q}_{\gamma}$ will preserve $\sigma_{\gamma}$ on $\mathbb{D}$, therefore $\sigma_{\gamma}$ is invariant under $\tilde{Q}_{\gamma}$ on all $\widehat{\mathbb{C}}$. Moreover, the map $\tilde{Q}_{\gamma}$ is holomorphic on $\widehat{\mathbb{C}} \backslash \overline{\mathbb{D}}$. Hence $\sigma_{\gamma}$ defines a Beltrami form $\mu$ with $\|\mu\|_{\infty}<1$.

By the Measurable Riemann Mapping Theorem [Ah, [L], there exists a unique quasiconformal homeomorphism $\psi_{\gamma}: \widehat{\mathbb{C}} \rightarrow \widehat{\mathbb{C}}$, normalized by $\psi_{\gamma}(\infty)=\infty, \psi_{\gamma}(1)=0$ and $\psi_{\gamma}\left(H_{p}^{-1}(0)\right)=\frac{e^{2 \pi i \gamma}}{2}$, such that $\psi_{\gamma}^{*} \sigma_{0}=\sigma_{\gamma}$. We define

$$
F_{\gamma}=\psi_{\gamma} \circ \tilde{Q}_{\gamma} \circ \psi_{\gamma}^{-1},
$$

then $F_{\gamma}$ is holomorphic. Since the map $\psi_{\gamma}$ fixes the point at infinity, we can consider $F_{\gamma}: \mathbb{C} \rightarrow \mathbb{C}$, which is a proper map of degree 2 . Thus $F_{\gamma}$ is a quadratic polynomial.

By construction, $F_{\gamma}$ is quasiconformally equivalent to the rotation $R_{\gamma}$ on $\psi_{\gamma}(\mathbb{D})$, therefore $\psi_{\gamma}(\mathbb{D})$ belongs to a Siegel disk $\Delta$ of $F_{\gamma}$. Since the orbit of zero under $F_{\gamma}$ is dense on the boundary of $\psi_{\gamma}(\mathbb{D})$, it follows that $\psi_{\gamma}(\mathbb{D})=\Delta$. Moreover, by the normalization of $\psi_{\gamma}$ there is a unique value $c(\gamma)$ such that $F_{\gamma}=P_{c(\gamma)}$. In summary, the following result holds.

Theorem 3.1 (Herman). Let $c \in M$ such that $P_{c}$ has a fixed Siegel disk $\Delta$ with rotation number $\gamma$. If $\gamma$ is of bounded type, then the boundary of $\Delta$ is a quasicircle that contains 0 .

To prove that the Julia set of $F_{\gamma}$ is locally connected, Petersen introduced an address system in the set of preimages of $\mathbb{D}$ under $\tilde{Q}_{\gamma}$.

3.2. Addresses of the drops. The address system that we are going to define on the components of the interior of $K\left(\tilde{Q}_{\gamma}\right)$, induces an address system in $\operatorname{int}\left(K_{c(\gamma)}\right)$ using the isomorphism $\psi_{\gamma}$ Pe, YaZa].

A drop is a component $U \subset \operatorname{int}\left(K\left(\tilde{Q}_{\gamma}\right)\right)$ and it has order $n$ if $U \in\left\{\tilde{Q}_{\gamma}^{-n}(\mathbb{D})\right\} \backslash \mathbb{D}$. 
Definition 3.1. If $\mathrm{U}$ is a drop of order $n$, we define:

(1) the center $c(U)$ of $U$ as the unique point that is sent by $\tilde{Q}_{\gamma}^{n}$ to $H_{p}^{-1}(0)$,

(2) the root of $U$ as the unique point $x(U) \in \partial U$ such that $\tilde{Q}_{\gamma}^{n-1}(x(U))=1$.

Note that there is only one component $U_{1}$ of order 1 , which is called 1-drop that has the point 1 as a root and $\overline{U_{1}} \cap \overline{\mathbb{D}}=\{1\}$. Moreover, the boundary is a real analytic Jordan curve except at the root point, where it forms an angle of $\pi / 3$. The same property is true for every component $V$ of higher order; that is, $\partial V$ is a real analytic curve except in $x(V)$, for all $n$.

Proposition 3.1 ( $\mathrm{YaZa}$ ). Let $U$ and $V$ be two drops of orders $m$ and $n$, respectively.

(1) If $m<n$ and $\bar{U} \cap \bar{V} \neq \emptyset$, then $\bar{U} \cap \bar{V}=x(V)$.

(2) If $p \in \bigcup_{n \geq 0}\left\{\tilde{Q}_{\gamma}^{-n}(1)\right\}$, then there exists a unique drop $U$ such that $p=$ $x(U)$.

If $U$ and $V$ satisfy property (1), we say that $U$ is the parent of $V$ or that $V$ is a child of $U$. By our definition, $\mathbb{D}$ is said to be of generation zero and all its children are of generation 1. In general, a drop $U$ is of generation $k$ if its parent is of generation $k-1$. We set $U_{0}=\mathbb{D}$. For $n \geq 1$, let $x_{n}=\tilde{Q}_{\gamma}^{-n+1}(1) \cap \overline{\mathbb{D}}$ and let $U_{n}$ be the $n$-drop with root $x_{n}$. Now, let us associate to every multi-index $i=i_{1} i_{2} \cdots i_{k}$ of positive integers, the $m$-drop $U$ of generation $k$ with root $x_{i_{1} \cdots i_{k}}$, where $m=i_{1}+\cdots+i_{k}$. Once we define an index for all drops of generation 1, we proceed by induction over $k$ to define the general case. Suppose that we have defined $x_{i_{1} \cdots i_{k}}$ for all multi-index $i=i_{1} i_{2} \cdots i_{k}$ of length $m-1$. Then, for an index $i=i_{1} i_{2} \cdots i_{j}$ such that $m=i_{1}+\cdots+i_{j}$, we define

$$
x_{i_{1} i_{2} \cdots i_{j}}= \begin{cases}\tilde{Q}_{\gamma}^{-1}\left(x_{\left(i_{1}-1\right) i_{2} \cdots i_{j}}\right) \cap \partial U_{i_{1} i_{2} \cdots i_{j-1}} & \text { if } i_{1}>1 \\ \tilde{Q}_{\gamma}^{-1}\left(x_{i_{2} \cdots i_{j}}\right) \cap \partial U_{i_{1} i_{2} \cdots i_{j-1}} & \text { if } i_{1}=1 .\end{cases}
$$

We denote by $U_{i_{1} i_{2} \cdots i_{k}}$, the component with root $x_{i_{1} i_{2} \cdots i_{k}}$ (Proposition 3.1).

\subsection{Limbs and chains.}

Definition 3.2. Let $U_{i_{1} i_{2} \cdots i_{k}}$ be a drop of order $n$. We define the limb $L_{i_{1} i_{2} \cdots i_{k}}$ as the closure of the union of $U_{i_{1} i_{2} \cdots i_{k}}$ and all its descendants

$$
L_{i_{1} i_{2} \cdots i_{k}}=\overline{\bigcup U_{i_{1} i_{2} \cdots i_{k} \cdots}}
$$

Note that $L_{0}=K\left(\tilde{Q}_{\gamma}\right)$. In order to have a useful partition of the filled Julia set $K\left(\tilde{Q}_{\gamma}\right)$, we expect that the boundary of a limb $\neq L_{0}$ is not the whole $J\left(\tilde{Q}_{\gamma}\right)$, which follows by the next key lemma of Petersen $[\mathrm{Pe}$.

Lemma 3.1 (Only two rays). Suppose that $0<\gamma<1$ is an irrational number. Then the critical point $z=1$ of $\tilde{Q}_{\gamma}$ is the landing point of two and only two external rays $R_{K}(t)$ and $R_{K}(s)$ in $A_{\gamma}(\infty)$.

Let $W_{1}$ denote the connected component of $\mathbb{C} \backslash\left(R_{K}(t) \cup R_{K}(s) \cup\{1\}\right)$ containing the drop $U_{1}$. We call $W_{1}$ the wake with root $x_{1}$. Given an arbitrary multi-index $i_{1} \cdots i_{k}$, we define the wake $W_{i_{1} \cdots i_{k}}$ as the appropriate pull-back of $W_{1}$. In fact, it follows that

$$
L_{i_{1} \cdots i_{k}}=\bar{W}_{i_{1} \cdots i_{k}} \cap K\left(\tilde{Q}_{\gamma}\right) .
$$


Proposition 3.2 (YaZa $)$. Consider $\tilde{Q}_{\gamma}$ for an irrational number $0<\gamma<1$. Then:

(1) If a drop $U$ is contained in the limb $L$, then all the children of $U$ are also contained in $L$.

(2) Any two limbs and any two wakes are either disjoint or nested.

(3) For every limb $L_{i_{1} i_{2} \cdots i_{k}}$, we have

$$
\tilde{Q}_{\gamma}\left(L_{i_{1} i_{2} \cdots i_{k}}\right)= \begin{cases}L_{\left(i_{1}-1\right) i_{2} \cdots i_{k}} & \text { if } i_{1}>1, \\ L_{i_{2} \cdots i_{k}} & \text { if } i_{1}=1 .\end{cases}
$$

Theorem 3.2 ( $\mathrm{Pe}, \overline{\mathrm{Ya}})$. If $\gamma \in \mathbb{T}$ is an irrational number, then

$$
\lim _{k \rightarrow \infty} \operatorname{Diam}\left(L_{i_{1} i_{2} \cdots i_{k}}\right)=0 .
$$

Corollary 3.1. Let $\gamma \in \mathbb{T}$ be an irrational number. For the Hausdorff topology,

$$
\lim _{k \rightarrow \infty} \overline{L_{i_{1} i_{2} \cdots i_{k}}}=\{p\} .
$$

Theorem $3.3(\underline{\mathrm{Pe}}, \underline{\mathrm{Ya}})$. Let $\gamma \in \mathbb{T}$ be an irrational number. Then $J\left(\tilde{Q}_{\gamma}\right)$ and $J\left(Q_{\gamma}\right)$ are locally connected.

Definition 3.3. Let $\left\{U_{0}=\mathbb{D}, U_{i_{1}}, U_{i_{1} i_{2}}, \ldots\right\}$ be a sequence of drops such that $U_{i_{1} i_{2} \cdots i_{k}}$ is the parent of $U_{i_{1} i_{2} \cdots i_{k+1}}$. We define a drop-chain as

$$
\mathcal{C}=\overline{\bigcup_{k} U_{i_{1} i_{2} \cdots i_{k}}}
$$

Consider the corresponding limbs

$$
K\left(\tilde{Q}_{\gamma}\right)=L_{0} \supset L_{i_{1}} \supset L_{i_{1} i_{2}} \supset L_{i_{1} i_{2} i_{3}} \supset \cdots
$$

which are nested by Proposition 3.2. The intersection of these limbs must be a unique point which we denote by $p(\mathcal{C})$ :

$$
p(\mathcal{C})=\bigcap_{k} L_{i_{1} i_{2} \cdots i_{k}} .
$$

By Corollary 3.1 for every chain $\mathcal{C}$, the point $p(\mathcal{C})$ is the limit point of $L_{i_{1} i_{2} \cdots i_{k}}$ as $k$ goes to infinity, with the Hausdorff topology. Thus,

$$
\mathcal{C}=\bigcup_{k} \bar{U}_{i_{1} i_{2} \cdots i_{k}} \cup\{p(\mathcal{C})\},
$$

is compact, connected and locally connected.

For every drop $U \subset \operatorname{int} K\left(\tilde{Q}_{\gamma}\right)$, we have defined its center $c(U)$. By a ray in a drop $U$ we mean a hyperbolic geodesic which connects some boundary point $p \in \partial U$ to the center $c(U)$. This ray is denoted by $[p, c(U)]=[c(U), p]$. For two distinct points $p, q \in \partial U$, we denote by $[p, q]$ the union of the ray $[p, c(U)]$ with the ray $[c(U), q]$.

Given any drop-chain $\mathcal{C}$, there exists a unique "shortest" path $R=R(\mathcal{C})$ in $\mathcal{C}$ which connects 0 to $p(\mathcal{C})$. In fact, if $\mathcal{C}$ is of the form $\overline{\bigcup_{k} U_{i_{1} i_{2} \cdots i_{k}}}$, we define

$$
R(\mathcal{C})=\left[0, x_{i_{1}}\right] \cup \bigcup_{k \geq 1}\left[x_{i_{1} \cdots i_{k}}, x_{i_{1} \cdots i_{k+1}}\right] \cup p(\mathcal{C}) .
$$

It is easy to see that $R(\mathcal{C})$ is a piecewise analytic embedded arc in the plane. We call $R(\mathcal{C})$ the drop-ray associated with $\mathcal{C}$. We often say that $R(\mathcal{C})$ or $\mathcal{C}$ lands at $p(C)$. 
Proposition 3.3 (YaZa $)$. Let $\gamma \in \mathbb{T}$ be an irrational number.

(1) If $x \in K\left(\tilde{Q}_{\gamma}\right)$, then either it belongs to the closure of an interior component $U$ of $K\left(\tilde{Q}_{\gamma}\right)$, or it is the limit point of a chain.

(2) The map $\mathcal{C} \mapsto p(\mathcal{C})$ is one-to-one.

\section{Mating's MOdeL}

The goal of this section is to describe the dynamics of a family of cubic rational maps that will be used as a model for the mating. This family leaves $\mathbb{T}$ invariant and every element of the family has two critical points of cubic type on $\mathbb{T}$. We will modify this family, using quasiconformal surgery, to obtain a family of quadratic rational maps, where one of its elements will be the mating of $P_{c}$ and $P_{c^{\prime}}$.

4.1. There is a good family. In this part we show the following proposition.

Proposition 4.1. There exists $C>0$ such that if $\gamma \in(0,1 / 2)$ is irrational, then there is a unique $\lambda=\lambda(\gamma) \in(-1, C)$ such that the rational map

$$
B_{\lambda}=\zeta \circ \xi_{\lambda} \circ \zeta^{-1}
$$

leaves $\mathbb{T}$ invariant, where

$$
\zeta(z)=\frac{z-i}{z+i} \quad \text { and } \quad \xi_{\lambda}(z)=\frac{\lambda z^{3}+1}{-z^{3}+1} .
$$

The rotation number of $\left.B_{\lambda}\right|_{\mathbb{T}}$ is $\gamma$ and $\left.B_{\lambda}\right|_{\mathbb{T}}$ is a critical circle map with two critical points at -1 and 1.

Proof. First, note the following properties of the map $\xi_{\lambda}$ :

(1) For all $\lambda \in \mathbb{R}, \xi_{\lambda}(\mathbb{R})=\mathbb{R}$.

(2) If $\lambda>-1$, then $\xi_{\lambda}$ is increasing on $(-\infty, 1)$ and on $(1, \infty)$.

(3) It has two critical points of cubic type at 0 and $\infty$.

Since $\zeta: \overline{\mathbb{H}} \longrightarrow \overline{\mathbb{D}}$ is a $\mathbb{C}$-analytic isomorphism and $\zeta(0)=-1$, using (1), (2) and (3), we have that for all $\lambda>-1$, the restriction of the map $B_{\lambda}$ to $\mathbb{T}$ is a homeomorphism with two critical points of cubic type at 1 and -1 .

It remains to show that there exists an interval contained in $[-1,+\infty)$ where $B_{\lambda}$ realizes all the rotation numbers. However, by the definition of a rotation number, we have the following.

Remark 4.1. Let $f: \mathbb{T} \longrightarrow \mathbb{T}$ be a homeomorphism. If there is $x \in \mathbb{T}$ such that $f^{2}(x)<x+1$, then the rotation number of $f$ is less than or equal to $1 / 2$.

We will look for an interval of the form $(-1, C)$ that realizes all the rotation numbers. Since -1 satisfies Remark 4.1, we cannot expect that $B_{\lambda}$ realizes rotation numbers greater than $1 / 2$.

First of all, we will find a value $\lambda_{0}$ such that $B_{\lambda_{0}}$ has rotation number 0 . This is equivalent to finding $\lambda$ for which $\xi_{\lambda}$ has a real fixed point. This is done by considering the roots of the polynomial

$$
p_{\lambda}(z)=z^{4}+\lambda z^{3}-z+1 .
$$

Since $p_{\lambda}^{\prime}(z)=4 z^{3}+3 \lambda z^{2}-1$ has only one real root $r_{0}$ when $\lambda \in(-1,0)$ and the degree of $p_{\lambda}(z)$ is even, we have that $p_{\lambda}(z)$ has a global minimum in $r_{0}$. Moreover, if $p_{\lambda}\left(r_{0}\right)>0$, then $\xi_{\lambda}$ does not have real fixed points for $\lambda \in(-1,0)$. Thus, there 
exists $\lambda_{0}>0$ minimum such that the polynomial $p_{\lambda_{0}}(z)$ has a root with multiplicity two; therefore,

$$
\lambda_{0}=\frac{2-a}{a^{3}}-2 a \text { where } a=(\sqrt[3]{-37+\sqrt{1377}}-\sqrt[3]{37+\sqrt{1377}}-1) / 3,
$$

$\lambda_{0} \cong 2.23407602$ and $\xi_{\lambda_{0}}$ has a negative fixed point $x_{0}$ with multiplicity 2 .

Since the rotation number of $B_{\lambda}$ is different from 0 for every $\lambda \in\left(-1, \lambda_{0}\right)$, by Proposition 2.1 and the following lemma, we complete the proof.

Lemma 4.1. The rotation number of $B_{\lambda}$ converges to $1 / 2$ as $\lambda$ goes to -1 by the right.

Proof. Since the rotation number is invariant under conjugation, we can conjugate the family $\xi_{\lambda}$ by the family of affine maps

to obtain

$$
M_{\lambda}(z)=\sqrt{\frac{\lambda+1}{3}} z+1,
$$

$$
\varphi_{\lambda}=M_{\lambda}^{-1} \circ \xi_{\lambda} \circ M_{\lambda}(z)=-\frac{\sqrt{3 \lambda+3}(\lambda+1) z^{3}+9(\lambda+1) z^{2}+9}{\left((\lambda+1) z^{2}+3 \sqrt{3 \lambda+3} z+9\right) z} .
$$

Then

$$
\lim _{\lambda \rightarrow-1^{+}} \varphi_{\lambda}(z)=-\frac{1}{z} .
$$

Since this limit is uniform in $\lambda$ and $\varphi_{-1}(z)=-\frac{1}{z}$ on $\overline{\mathbb{R}}$, it has a periodic point of period 2 and the rotation number of $B_{\lambda}$ goes to $1 / 2$ when $\lambda$ goes to -1 .

4.2. Quasi-conformal surgery for $B_{\lambda}$. Let us fix $\gamma \in(0,1 / 2)$ and set $B_{\gamma}=$ $B_{\lambda(\gamma)}$.

Lemma 4.2. If $\gamma$ is an irrational number, then $J\left(B_{\gamma}\right)=\widehat{\mathbb{C}}$.

Proof. By Sullivan's theorem on non-wandering domains, it is sufficient to show that $B_{\gamma}$ does not have attracting cycles, parabolic cycles or rotation domains (Siegel disks or Herman rings) $\mathrm{B}, \mathrm{CG}, \mathrm{S}$.

Since $\gamma$ is irrational, the orbits under $B_{\gamma}$ of -1 and 1 are dense in $\mathbb{T}$, and since 1 and -1 are the only critical points, $B_{\gamma}$ cannot have attracting cycles, parabolic cycles or Herman rings [F, D2]. On the other hand, $B_{\gamma}$ cannot have Siegel disks since the boundary of a Siegel disk is contained in the closure of the critical orbit and the sets $\mathbb{D}, \mathbb{C} \backslash \mathbb{D}$ are not invariant under $B_{\gamma}$.

By Yoccoz's Theorem 2.1, if $\gamma$ is irrational, there exists a homeomorphism $h$ : $\mathbb{T} \rightarrow \mathbb{T}$ that conjugates $B_{\gamma}$ to $R_{\gamma}$. Moreover, if $\gamma$ is of bounded type and $h(1)=1$, by Herman-Świątek's Theorem 2.2 there is a unique quasisymmetric homeomorphism $h$ that realizes the conjugation. By Douady-Earle's Theorem, $h$ can be extended as a quasiconformal map $\mathrm{H}$ of the unit disk (see $[\mathrm{DE}]$ ). We define,

$$
\tilde{B}_{\gamma}= \begin{cases}B_{\gamma}(z), & |z|>1, \\ H^{-1} \circ R_{\gamma} \circ H(z), & |z| \leq 1 .\end{cases}
$$

This new map has two critical points at 1 and -1 of order 2 and it is holomorphic on the complement of the unit disk.

Now, we define on $\widehat{\mathbb{C}}$ a new conformal structure invariant under $\tilde{B}_{\gamma}$. Let $\sigma_{0}$ be the standard structure of $\widehat{\mathbb{C}}$. On $\mathbb{D}$, we define $\sigma_{\gamma}=H^{*}\left(\sigma_{0}\right)$ and on every drop 
$U_{n} \neq \mathbb{D}$ of $\tilde{B}_{\gamma}^{-n}(\mathbb{D})$, we define $\sigma_{\gamma}=\left(\tilde{B}_{\gamma}^{\circ n}\right)^{*}\left(\left.\sigma_{\gamma}\right|_{\mathbb{D}}\right)$. Finally, we define $\sigma_{\gamma}=\sigma_{0}$ on the complement of $\bigcup_{n \geq 0} \tilde{B}_{\gamma}^{-n}(\mathbb{D})$ (by Lemma 4.2 it has an empty interior).

By construction, $\sigma_{\gamma}$ is invariant under $\tilde{B}_{\gamma}$ on all $\widehat{\mathbb{C}}$. Since the map $\tilde{B}_{\gamma}$ is holomorphic on $\widehat{\mathbb{C}} \backslash \overline{\mathbb{D}}, \sigma_{\gamma}$ defines a Beltrami form $\mu$ and $\|\mu\|_{\infty}<1$.

By the Measurable Riemann Mapping Theorem [Ah, LV], there exists a unique quasiconformal homeomorphism $\varsigma_{\gamma}: \widehat{\mathbb{C}} \rightarrow \widehat{\mathbb{C}}$ normalized by $\varsigma_{\gamma}(1)=1, \varsigma_{\gamma}(-1)=-1$ and $\varsigma_{\gamma}\left(H^{-1}(0)\right)=0$, such that $\varsigma_{\gamma}^{*} \sigma_{0}=\sigma_{\gamma}$. We define

$$
F_{\gamma}=\varsigma_{\gamma} \circ \tilde{B}_{\gamma} \circ \varsigma_{\gamma}^{-1}
$$

then $F_{\gamma}$ is holomorphic, $F_{\gamma}(0)=0$ and it has a domain $\varsigma_{\gamma}(\mathbb{D})$ that contains 0 , where it is conjugated to $R_{\gamma}$. Since the orbit of -1 is dense in the boundary of $\varsigma_{\gamma}(\mathbb{D})$, the Siegel disk $\Delta$ of $F_{\gamma}$ in 0 is equal to $\varsigma_{\gamma}(\mathbb{D})$. Thus, if $\eta=e^{2 \pi i \gamma}$, there exists a unique $a(\eta) \in \mathbb{C}$ such that

$$
F_{\gamma}=R_{\eta, a(\eta)} .
$$

Hence, we have the following result.

Theorem 4.1. If $\gamma$ is an irrational number of bounded type and $\eta=e^{2 \pi i \gamma}$, then there exists a unique value $a(\eta)$ such that $R_{\eta, a(\eta)}$ has a fixed Siegel disk $\Delta$ whose boundary is a quasicircle that contains $1,-1$ and it verifies $R_{\eta, a(\eta)}^{2}(-1)=1$.

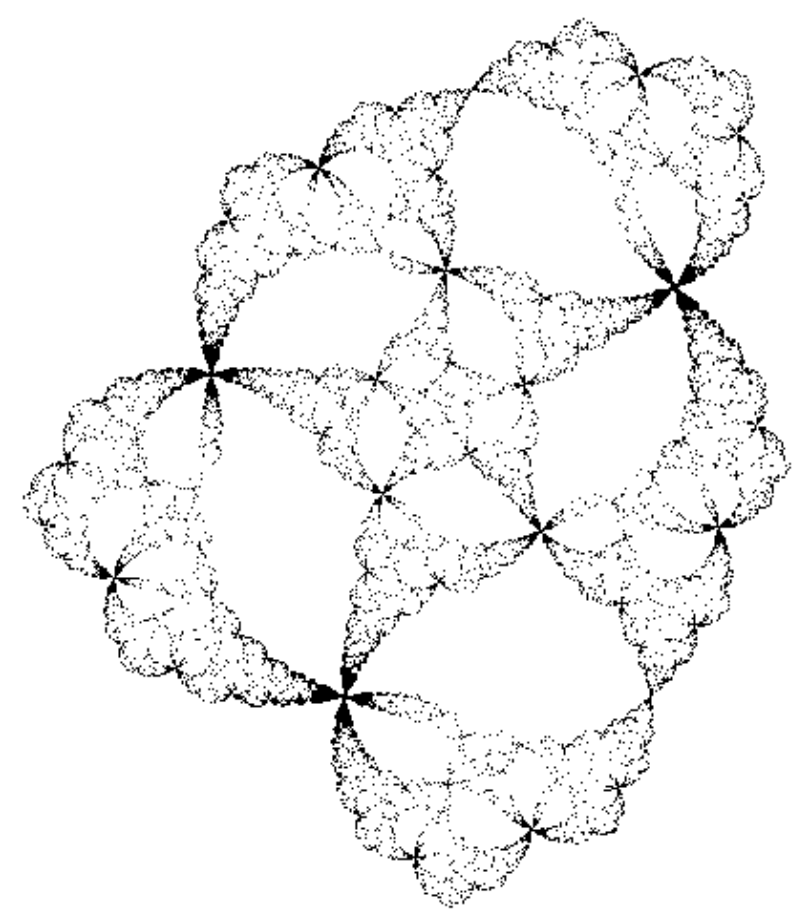

FiguRE 1. Fatou set of $R_{\eta, a(\eta)}$ with $\eta=e^{2 \pi i \gamma}$ and $\gamma=[2,1,1,1, \ldots]$ 
The value $a(\eta)$ can be calculated from the condition

$$
R_{\eta, a(\eta)}^{2}(-1)=1
$$

This equation has two solutions,

$$
a(\eta)=\frac{1}{2} \frac{\eta^{2}+4+\sqrt{\eta^{4}-8 \eta^{3}}}{\eta+1}
$$

and its complex conjugated $c(\eta)$. One can discard $c(\eta)$, since the boundary of the Siegel disk associated to $R_{\eta, c(\eta)}$ does not contain -1 which is the other critical point (see Figure 1).

\section{AdDress in the MATing'S MODEL}

We will define on the components of $\tilde{B}_{\gamma}^{-n}(\mathbb{D})$, a similar address system to the one we have defined for Petersen's model.

First, we recall that a drop $U$ has order $n$, or it is an $n$-drop if it is a component of $\tilde{B}_{\gamma}^{-n}(\mathbb{D}) \backslash \mathbb{D}$.

Definition 5.1. Let $U$ be an $n$-drop. We define the following:

(1) the root of $U$ as the unique point $x(U) \in \partial U$ such that $\tilde{B}_{\gamma}^{n-1}(x(U))=1$;

(2) the pre-root of $U$ as the unique point $y(U) \in \partial U$ such that $\tilde{B}_{\gamma}^{n-1}(y(U))$ $=-1$;

(3) the center of $U$ as $c(U)=\tilde{B}_{\gamma}^{-n}\left(H^{-1}(0)\right) \in U$.

We set $U_{0}=\mathbb{D}$ and let $U_{1}$ be the 1-drop, that is, the immediate preimage of $\mathbb{D}$ and $\bar{U}_{1} \cap \overline{\mathbb{D}}=\{1,-1\}$. The problem that appears in the study of the set of components of $\tilde{B}_{\gamma}^{-n}(\mathbb{D})$ is that the roots do not determine, in a unique way, the components. For instance, the point -1 is the root of at least two components. In order to associate an index to each component, we will need some additional definitions. Let

$$
\begin{aligned}
\mathbf{R}_{\gamma}=\{p \in \mathbb{C}: & \text { if } \left.\tilde{B}_{\gamma}^{n}(p)=1 \text { for } n \geq 0\right\}, \\
\mathbf{P R}_{\gamma}=\{p \in \mathbb{C}: & \text { if } \left.\tilde{B}_{\gamma}^{n}(p)=-1 \text { for } n \geq 0\right\} .
\end{aligned}
$$

Proposition 5.1. The following holds:

(1) If $p \in \mathbf{R}_{\gamma} \backslash \mathbf{P} \mathbf{R}_{\gamma}$, then there exists a unique drop $U$ that has root $p$.

(2) If $p \in \mathbf{P R}_{\gamma}$, there exist two and only two drops with root $p$.

Proof. (1) If $p \in \mathbf{R}_{\gamma} \backslash \mathbf{P} \mathbf{R}_{\gamma}$, then the first time that an iterate of $p$ hits the boundary of the unit disk is at the point 1 . Hence, it is sufficient to show that there exists a unique drop with root 1 . Suppose that there is another drop $U \neq U_{1}$ of order $n>1$, with 1 as a root point. Then,

$$
\tilde{B}_{\gamma}^{n-1}(U)=U_{1} \text { implies } \tilde{B}_{\gamma}^{n-1}(1)=1 .
$$

This is a contradiction since $\gamma$ is irrational.

(2) Since $\gamma$ is irrational, $1 \notin \mathbf{P} \mathbf{R}_{\gamma}$, and since $\tilde{B}_{\gamma}$ is a local isomorphism for every point $p \in \mathbf{P R}_{\gamma} \backslash\{-1\}$, it is sufficient to show that there are only two components with root point -1 .

We recall that $\tilde{B}_{\gamma}^{\circ 2}(-1)=1$ and that there is only one component $U_{2}$ of order two with root point $-i$. Since $-i$ is a critical value, there are two components $U_{3}, U_{3}^{\prime}$ of order 3 with root -1 , which are mapped on $U_{2}$ in one iteration and that finally arrive to $U_{1}$ after two iterations. 
Suppose that there is another drop $U$ of order $n>3$ with root point -1 . Then

$$
\tilde{B}_{\gamma}^{\circ n-2}(U)=\tilde{B}_{\gamma}\left(U_{3}\right)=U_{2} \text { implies } \tilde{B}_{\gamma}^{\circ n-2}(-1)=\tilde{B}_{\gamma}(-1) .
$$

We obtain a contradiction since $\tilde{B}_{\gamma}$ has no periodic points in $\mathbb{T}$.

Corollary 5.1. Let $U$ and $V$ be two drops of order $n$ and $m$, respectively.

(1) If $m=n>2$ and $\partial U \cap \partial V \neq \emptyset$, then $\partial U \cap \partial V=x(U)=x(V)$ and there exists a drop $W$ of order $m-2$ such that $y(W)=x(U)$.

(2) If $m>n$, and $\partial U \cap \partial V \neq \emptyset$, then $\partial U \cap \partial V \subset\{x(V), y(V)\}$.

Remark 5.1. We have the following properties:

(1) If we give a positive oriented parametrization $J:[0,1] \rightarrow \mathbb{T}$ of $\mathbb{T}$, such that $J(0)=1$ and $J\left(\frac{1}{2}\right)=-1$, it induces an orientation in the boundary of any drop.

(2) The boundary of $U_{1}$ is a real analytic Jordan curve, except at the points 1 and -1 , where it forms an angle of $\pi / 3$ with the boundary of the disk.

(3) The parametrization induced in the boundary of the component $U$ of order $n$, sends 0 in $x(U), 1 / 2$ in $y(U)$ and the boundary is $\mathbb{R}$-analytic on $(0,1) \backslash\{1 / 2\}$.

Definition 5.2. Let $U$ and $V$ be two drops of order $n$ and $m$, respectively, with $m>n$. If $x(V) \in \partial U$, we define the angle of tangency between $V$ and $U$ at the point $x(V)$ as the angle between the two tangents $T_{U}(x(V))$ and $T_{V}(x(V))$, where

$$
T_{U}(x(V))=\lim _{t \rightarrow t_{0}^{-}} J_{U}^{\prime}(t) \text { and } T_{V}(x(V))=\lim _{t \rightarrow 0^{+}} J_{V}^{\prime}(t)
$$

and $t_{0}$ is such that $J_{U}\left(t_{0}\right)=x(V)$.

Definition 5.3. Let $U$ and $V$ be two drops of order $n$ and $m$, respectively, with $m>n$. We say that $U$ is the parent of $V(V$ is the child of $U)$ if $x(V) \in \partial U$ and the angle of tangency between $V$ and $U$ at the point $x(V)$ is equal to $\pi / 3$.

Since $\tilde{B}_{\gamma}$ is holomorphic and it does not have critical points in $\widehat{\mathbb{C}} \backslash \overline{\mathbb{D}}$, it preserves the angles and therefore every drop has a unique parent. Since we want to associate a multi-index $i_{1} \cdots i_{k}$ to every component $U$, we will proceed in the following way. First, we denote by $U_{m}$ the unique $m$-drop whose parent is $\mathbb{D}$. Next, we define, in a recurrent way, the index for all $n$-drops.

Suppose that we have associated to every component of order $k<n$, a multiindex $i_{1} \cdots i_{k}$. If $U$ is a component of order $n$, then its parent $V$ is a component of order less than $n$, then there exists $k$ such that $V=U_{i_{1} \cdots i_{k-1}}$. We associate to $U$ the multi-index $i_{1} \cdots i_{k}$; that is, $U=U_{i_{1} \cdots i_{k}}$ where $i_{k}=n-\sum_{j=1}^{k-1} i_{j}$.

We deduce directly,

$$
\tilde{B}_{\gamma}\left(U_{i_{1} i_{2} \cdots i_{k}}\right)= \begin{cases}U_{\left(i_{1}-1\right) i_{2} \cdots i_{k}} & \text { if } i_{1}>1 \\ U_{i_{2} \cdots i_{k}} & \text { if } i_{1}=1 .\end{cases}
$$

Proposition 5.2. Let $U=\mathbb{D}$ and let $V$ be a drop such that $\partial U \cap \partial V=\{x(V), y(V)\}$.

(1) If $\gamma<1 / 3$, then $V=U_{1}$.

(2) If $1 / 3<\gamma<1 / 2$, then $V$ is equal to either $U_{1}, U_{2}$ or $U_{12}$.

Proof. (1) If $\gamma<1 / 3$, the preimage of -1 under $\tilde{B}_{\gamma}$ that belongs to $\mathbb{T}$ is in the upper half plane and the other preimage, that is, the pre-root of $U_{2}$, is on the boundary of $U_{1}$. Therefore $\partial U_{2}$ does not intersect $\mathbb{T}$, except at $-i$. If $U$ is a drop 


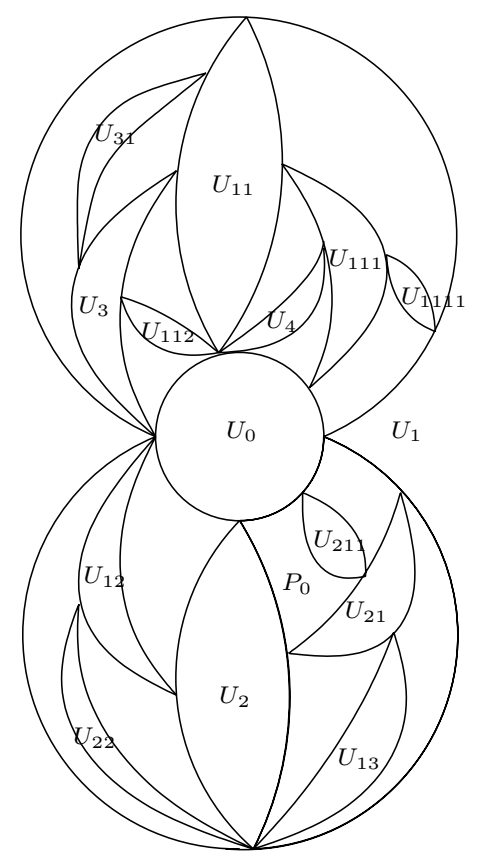

(1)

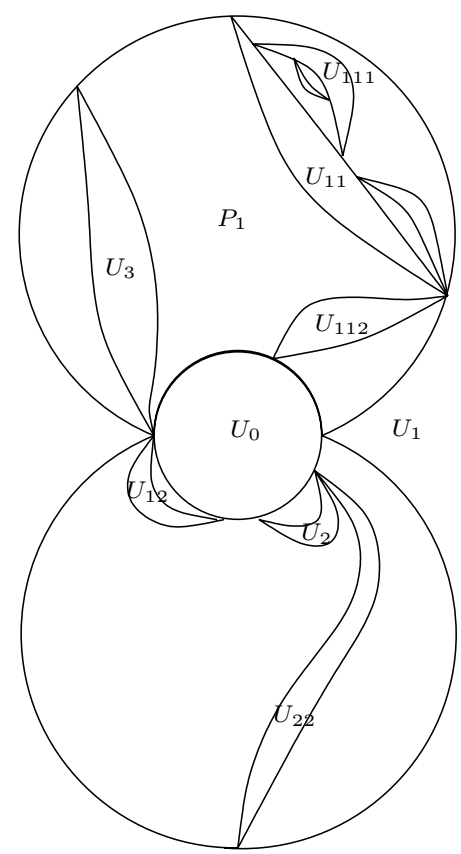

$(2)$

Figure 2. (1) shows the first address for $\gamma<\frac{1}{3}$ and (2) for $\gamma>\frac{1}{3}$

different from $U_{1}$ and $\bar{U} \cap \mathbb{D}=\{x(U), y(V)\}$, then all the iterates of $U$ intersect $\mathbb{T}$ in two points. In particular, there exists $n \in \mathbb{N}$ such that $U_{2}=\tilde{B}_{\gamma}^{n}(U)$. But this is a contradiction, since $\partial U_{2} \cap \mathbb{T}=\{-i\}$.

(2) By the same argument as in (1), it is sufficient to show that the boundary of a component of order $n>3$ cannot intersect $\mathbb{T}$ except in one point (see Figure 22).

When $\gamma>1 / 3$, the preimage of -1 is on the lower half plane, hence the drop $U_{2}$ intersects $\mathbb{T}$ at the points $x\left(U_{2}\right)$ and $y\left(U_{2}\right)$. The same happens for $U_{12}$, which is the drop with root -1 that is contained in the lower half plane. However, there are no drops of period 4 whose boundary intersects $\mathbb{T}$ in two points, because the only two candidates are the preimages of $U_{12}$; that is, $U_{112}$ and $U_{22}$, but they are different, and the boundary of each of them cannot intersect $\mathbb{T}$ in two points, since the preimages under $\tilde{B}_{\gamma}$ of $x\left(U_{12}\right)$ and $y\left(U_{12}\right)$, which are $x_{-3}$ and $x_{-5}$, respectively, belong to two different half planes.

Definition 5.4. Let $U_{i_{1} i_{2} \cdots i_{k}}$ be a drop of order $n$. We define the limb $L_{i_{1} i_{2} \cdots i_{k}}^{\prime}$ as the closure of the union of $U_{i_{1} i_{2} \cdots i_{k}}$ and all its descendants

$$
L_{i_{1} i_{2} \cdots i_{k}}^{\prime}=\overline{\bigcup U_{i_{1} i_{2} \cdots i_{k} \cdots}}
$$

By definition we have the following properties. 
Proposition 5.3. The following holds:

(1) If $U$ is a drop contained in a limb $L^{\prime}$, then every child of $U$ is also contained in $L^{\prime}$.

(2) If $L_{1}^{\prime}, L_{2}^{\prime}$ are two limbs, then they are either disjoint or nested.

(3) We have, for every limb, $L_{i_{1} i_{2} \cdots i_{k}}^{\prime}$,

$$
\tilde{Q}_{\gamma}\left(L_{i_{1} i_{2} \cdots i_{k}}^{\prime}\right)= \begin{cases}L_{\left(i_{1}-1\right) i_{2} \cdots i_{k}}^{\prime} & \text { if } i_{1}>1, \\ L_{i_{2} \cdots i_{k}}^{\prime} & \text { if } i_{1}=1 .\end{cases}
$$

Definition 5.5. Let $\left\{U_{0}=\mathbb{D}, U_{i_{1}}, U_{i_{1} i_{2}}, \ldots\right\}$ be a sequence of drops such that $U_{i_{1} i_{2} \cdots i_{k}}$ is the parent of $U_{i_{1} i_{2} \cdots i_{k+1}}$. We define a drop-chain as

$$
\mathcal{C}=\overline{\bigcup_{k} U_{i_{1} i_{2} \cdots i_{k}}} \text {. }
$$

In the following section, we will show that the limit of $L_{i_{1} i_{2} \cdots i_{k}}^{\prime}$ exists when $k$ goes to infinity. In fact, we will prove an equivalent result to Theorem 3.2 .

\section{LOCAL CONNECTIVITY}

The goal of this section is to prove the following result.

Theorem 6.1. If $\gamma \in \mathbb{T}$ is an irrational number, then

$$
\lim _{k \rightarrow \infty} \operatorname{Diam}\left(\overline{L_{i_{1} i_{2} \cdots i_{k}}^{\prime}}\right)=0 .
$$

By this theorem, we have that $p(\mathcal{C})=\bigcap_{k} L_{i_{1} i_{2} \cdots i_{k}}^{\prime}$ is a single point and we will say that $R(\mathcal{C})$ or $\mathcal{C}$ lands at $p(\mathcal{C})$.

6.1. Puzzle pieces of $\tilde{B}_{\gamma}$. We consider $0<\gamma<1 / 2$ irrational, and we denote by $\frac{p_{n}}{q_{n}}$ the $n$th convergent of the continued fraction of $\gamma$. Let $J_{\gamma}=J\left(\tilde{B}_{\gamma}\right)$ and $x_{k}=\left.\tilde{B}_{\gamma}^{k}\right|_{\mathbb{T}}(1)$, for all $k \in \mathbb{Z}$.

We define the puzzle pieces of level zero, $Y_{1}^{(0)}, Y_{2}^{(0)}, Y_{3}^{(0)}, Y_{4}^{(0)}$, as the components of $\mathbb{C} \backslash\left\{\mathbb{D} \cup U_{1} \cup U_{2} \cup U_{11}\right\}$. Then, we define the pieces of level $n, Y_{j}^{(n)}$ as the components of $\tilde{B}_{\gamma}^{-n}\left(Y_{i}^{(0)}\right)$ for $i=1,2,3,4$.

We define $P_{n}$, the critical pieces around 1 , in the following way. Let $P_{0}$ be the puzzle piece of level 0 that contains 1 and $x_{q_{1}}$. We inductively define $P_{n}$ as the closed set which is mapped homeomorphically onto $P_{n-1}$ by $\tilde{B}_{\gamma}^{q_{n}}$ and which contains 1 and $x_{q_{n+1}}$ (see Figure 3).

Remark 6.1. The following properties hold.

(1) The boundary of the piece $Y_{j}^{(n)}$ of level $n$ is contained in the union of the boundaries of the components of order $k \leq n+2$.

(2) For all $n, J_{\gamma} \subset \bigcup_{j} Y_{j}^{(n)}$.

(3) $Y_{j}^{(n)}$ is bounded for all $j, n \in \mathbb{N} \cup\{0\}$.

In what follows, to obtain a univalent preimage of the puzzle piece $P_{0}$, we use the holomorphic inverse branches of $\tilde{B}_{\gamma}$. These preimages have the following nesting property.

Lemma 6.1. Let $A_{1}$ and $A_{2}$ be two distinct univalent preimages of the puzzle piece $P_{0}$ such that int $A_{1} \cap \operatorname{int} A_{2} \neq \emptyset$. Then, either $A_{1} \subset A_{2}$ or $A_{2} \subset A_{1}$. 


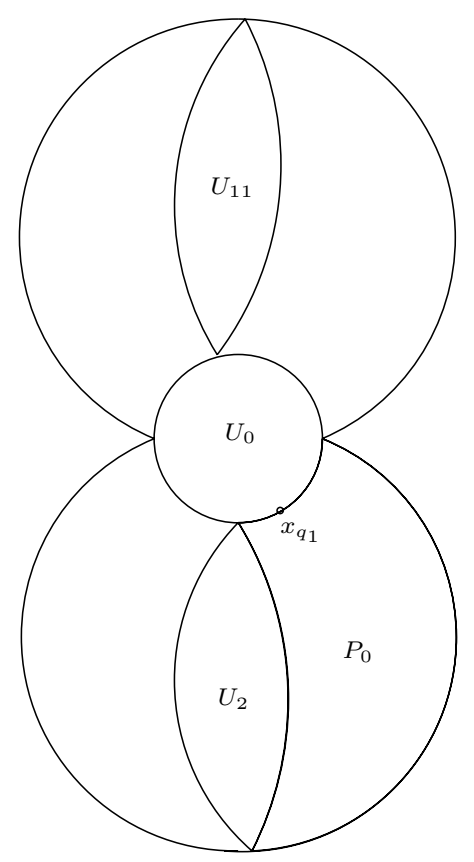

(1)

Figure 3. First piece of the puzzle $P_{0}$

Proof. Suppose that $A_{1} \cap A_{2} \neq \emptyset$ where $A_{1}$ is not contained in $A_{2}$ and $A_{2}$ is not contained in $A_{1}$. Let $\gamma=\partial P_{0}, \gamma_{1}=\partial A_{1}$ and $\gamma_{2}=\partial A_{2}$. Then $\gamma_{1} \cap \operatorname{int} A_{2} \neq \emptyset$ and $\gamma_{1} \cap \mathbb{C} \backslash \bar{A}_{2} \neq \emptyset$. We can assume that there exist $m$ and $n$, with $m \leq n$ such that $A_{1}=\tilde{B}_{\gamma}^{-m}\left(P_{0}\right)$ and $A_{2}=\tilde{B}_{\gamma}^{-n}\left(P_{0}\right)$. Since $\gamma_{2} \cap \operatorname{int} A_{1} \neq \emptyset$ and $\gamma_{2} \cap \mathbb{C} \backslash \bar{A}_{1} \neq \emptyset$, we have that $\tilde{B}_{\gamma}^{m}\left(\gamma_{2}\right) \cap \operatorname{int} P_{0} \neq \emptyset$ and $\tilde{B}_{\gamma}^{m}\left(\gamma_{2}\right) \cap \operatorname{int}\left(\mathbb{C} \backslash P_{0}\right) \neq \emptyset$.

Since $\tilde{B}_{\gamma}^{m}\left(\gamma_{2}\right) \cap \gamma \subset \gamma, \tilde{B}_{\gamma}^{m}\left(\gamma_{2}\right)$ should intersect $\gamma$ in a drop with root $x$ in $\overline{\mathbb{D}}, \bar{U}_{1}$ or $\bar{U}_{2}$. Under the function $\tilde{B}_{\gamma}^{\circ n-m}$ a small subarc $\tilde{\gamma}$ of $\tilde{B}_{\gamma}^{\circ m}\left(\gamma_{2}\right)$ containing $x$ is mapped homeomorphically to a subarc $\delta$ around $\tilde{B}_{\gamma}^{\circ n-m}(x)$. Since $x, \tilde{B}_{\gamma}(x), \ldots, \tilde{B}_{\gamma}^{\circ n-m}(x)$ do not pass through 1 , we have that $\delta$ intersects $\gamma$ transversally in $\tilde{B}_{\gamma}^{\circ n-m}(x)$, which is impossible since $\tilde{B}_{\gamma}^{\circ n}\left(A_{2}\right)=P_{0}$.

By the definition of puzzle piece and the previous lemma, we deduce the following result.

Proposition 6.1. Let $Y_{i}^{(n)}$ and $Y_{j}^{(m)}$ be two puzzle pieces such that int $Y_{i}^{(n)} \cap$ $\operatorname{int} Y_{j}^{(m)} \neq \emptyset$.

(1) If $m=n$, then $Y_{i}^{(n)}=Y_{j}^{(m)}$.

(2) If $m \neq n$, then $Y_{i}^{(n)} \subset Y_{j}^{(m)}$ or $Y_{j}^{(m)} \subset Y_{i}^{(n)}$.

Proposition 6.2. The following statements hold.

(1) For all $n$ and $j, Y_{j}^{(n)} \cap J_{\gamma}$ is connected.

(2) If $U_{i_{1} \cdots i_{k}} \subset \operatorname{int} Y_{j}^{(n)}$, then $L_{i_{1} \cdots i_{k}}^{\prime} \subset \operatorname{int} Y_{j}^{(n)}$. 
Proof. The first part follows by Proposition 6.1 and Remark 6.1. For the second part, suppose that there exists a drop $U_{i_{1} \cdots i_{k} \cdots i_{k+m_{0}}} \subset L_{i_{1} \cdots i_{k}}^{\prime}$ such that $U_{i_{1} \cdots i_{k} \cdots i_{k+m_{0}}}$ is not contained in int $Y_{j}^{(n)}$. Let $\mathcal{C}$ be the drop-chain associated to the following sequence of drops

$$
U_{i_{1} \cdots i_{k}}, U_{i_{1} \cdots i_{k} i_{k+1}}, \ldots, U_{i_{1} \cdots i_{k} \cdots i_{k+m_{0}}}, \ldots
$$

in the limb $L_{i_{1} \cdots i_{k}}^{\prime}$. Then, the ray $R(\mathcal{C})$ intersects the boundary of int $Y_{j}^{(n)}$ transversally. Since $\operatorname{int} Y_{j}^{(n)}$ is an univalent pull-back of $P_{0}$, there exists $m \in \mathbb{N}$ such that $\tilde{B}_{\gamma}^{\circ m}\left(\operatorname{int} Y_{j}^{(n)}\right)=P_{0}$. Hence, $\tilde{B}_{\gamma}(R(\mathcal{C}))$ intersects the boundary of $P_{0}$ transversally, which is a contradiction.

By Proposition 6.2, it is sufficient to show that the diameter of the pieces $Y_{j}^{(n)}$ goes to 0 as $n$ goes to infinity in order to prove Theorem 6.1.

Proposition 6.3. If $n \geq 1$, the following holds.

(1) $P_{n} \cap \partial \mathbb{D}=\left[1, x_{-q_{n}}\right]$.

(2) $\tilde{B}_{\gamma}^{\circ q_{n}}\left(P_{n}\right)=P_{n-1}$.

(3) $\tilde{B}_{\gamma}^{\circ q_{n}}\left(P_{n} \cap \partial U_{1}\right)=\left[x_{q_{n}}, x_{-q_{n-1}}\right]$.

(4) $P_{n}$ contains the component $U_{q_{n+2}+1}$.

Proof. By definition, $P_{1}$ is the piece of order $q_{1}$ that belongs to $\overline{\mathbb{H}}$ and contains 1 . We have that $x_{-q_{1}}$ is the closest preimage to 1 of order $k \leq q_{1}$, and $x_{q_{2}} \in\left[x_{-q_{1}}, 1\right]$, then $P_{1} \cap \partial \mathbb{D}=\left[x_{-q_{1}}, 1\right]$. Since $x_{-q_{n}}$ is the closest preimage to 1 of order $k \leq q_{n}$, we have (1).

Since $\tilde{B}_{\gamma}^{q_{n}}:\left(x_{-q_{n}}, x_{-q_{n}-q_{n-1}}\right) \rightarrow\left(x_{-q_{n-1}}, 1\right)$ is a homeomorphism, $\tilde{B}_{\gamma}^{-q_{n}}$ sends $P_{n-1}$ on $P_{n}$ and the $\operatorname{arc}\left[1, x_{-q_{n}}\right]$ on the union of the arc $\left[1, x_{-q_{n}}\right]$ and one arc of $\partial U_{1}$, we deduce (2) and (3). Since $\partial U_{q_{n+2}+1} \cap \overline{\mathbb{D}}=x_{-q_{n+2}} \in\left[1, x_{-q_{n}}\right]$, then (4) holds.

Since the intersection of $P_{n+2}$ with $\mathbb{T}$ is contained properly in $P_{n} \cap \mathbb{T}$, by Lemma 6.1 and Proposition 6.3 it follows that:

Corollary 6.1. For all $n \geq 0$ we have $P_{n+2} \subsetneq P_{n}$.

6.2. Complex bounds. Let us fix an irrational number $\gamma \in(0,1 / 2)$ of bounded type, and set $B=B_{\lambda(\gamma)}, \tilde{B}=\tilde{B}_{\lambda(\gamma)}$ and $\xi=\xi_{\lambda}$. Recall that $B_{\lambda}=\zeta \circ \xi_{\lambda} \circ \zeta^{-1}$, where

$$
\zeta(z)=\frac{z-i}{z+i} \quad \text { and } \quad \xi_{\lambda}(z)=\frac{\lambda z^{3}+1}{-z^{3}+1} .
$$

We define the following Möbius transformation that sends the upper-half plane into the interior of the unit disk

$$
\psi(z)=\frac{z+1-i}{-i z+1-i}
$$

and set $\varphi=\psi^{-1}$.

Set $B(1)=e^{2 \pi i \tau}$ with $0<\tau<1 / 2$. Observe that

$$
\frac{B(z)-B(1)}{(z-1)^{3}}
$$


is a bounded holomorphic function in the domain $\mathbb{C} \backslash \overline{\left(\mathbb{D} \cup U_{1}\right)}$. Thus, there exists some positive constant $C$ such that

$$
C^{-1}|z-1|^{3}<|B(z)-B(1)|<C|z-1|^{3}
$$

in this domain. We denote $S=\mathbb{C} \backslash \overline{\varphi\left(U_{1}\right)}$ and by $S_{J}$ the domain obtained by removing from $S$ the points of the real line that do not belong to the open interval $J \subset \mathbb{R}$,

$$
S_{J}=(S \backslash \mathbb{R}) \cup J .
$$

Let us fix $n \geq 2$ and consider the backward orbit of open intervals

$$
\left(1, B^{\circ q_{n}}(1)\right),\left(B^{-1}(1), B^{\circ q_{n}-1}(1)\right), \ldots,\left(B^{-q_{n}}(1), 1\right) .
$$

Let us set $\phi=\psi^{-1} \circ B_{\lambda}^{-1} \circ \psi$, where we choose the corresponding branch of $\xi^{-1}$ in such a way that the preimage of a real interval under the map $z^{3}$ is contained in the real line. Set $J_{-i}=\varphi\left(\left(B^{-i}(1), B^{\circ q_{n}-i}(1)\right)\right)$ and consider the $\phi$ orbit

$$
J_{0}, J_{-1}, J_{-2}, \ldots, J_{-q_{n}} \text {. }
$$

Using the combinatorics of closest returns (see section [2.2), it is not hard to see that $B^{\circ 2}(1) \notin\left(B^{-k}(1), B^{\circ q_{n}-k}(1)\right)$ for $0 \leq k \leq q_{n}$. By its definition, the map $\phi: J_{-k} \rightarrow J_{-k-1}$ for $0 \leq k \leq q_{n}-1$ has univalent extension to $S_{J_{-k}}$ and the range of this univalent map is a subset of $S_{J_{-k-1}}$, hence the composition $\phi^{\text {ol }}: J_{-k} \rightarrow J_{-k-l}$ for $0 \leq k<k+l \leq q_{n}$ univalently extends to the entire $S_{J_{-k}}$.

Consider the univalent extensions of the iterates $\phi^{\circ k}: J_{0} \rightarrow J_{-k}$ to the region $S_{J_{0}}$ for $1 \leq k \leq q_{n}$. Applying these univalent branches to a point $z \in S_{J_{0}}$, we obtain the backward orbit of $z$ corresponding to the orbit (4):

$$
z=z_{0}, z_{-1}, z_{-2}, \ldots, z_{q_{n}}, \text { where } z_{-k}=\phi^{\circ k}\left(z_{0}\right) .
$$

A corresponding backward orbit of a subset of $S_{J_{0}}$ is similarly defined.

Let $\mathbb{C}_{J} \supset S_{J}$ denote the slit plane $(\mathbb{C} \backslash \mathbb{R}) \cup J$. Since there is a conformal mapping of this domain to the upper-half plane, it is easy to verify that the hyperbolic neighborhood $\left\{z \in \mathbb{C}_{J}: \operatorname{dist}_{\mathbb{C}_{J}}(z, J)<r\right\}$ for $r>0$ is the union $D_{\theta}(J)$ of two Euclidean disks of equal radii with common chord $J$ intersecting the real axis at an outer angle $\theta=\theta(r) \underline{\mathrm{dMvS}}$. In this case, a computation yields

$$
r=\log \tan \left(\frac{\pi}{2}-\frac{\theta}{4}\right) \text {. }
$$

A standard argument shows that the hyperbolic neighborhood

$$
\left\{z \in S_{J}: \operatorname{dist}_{S_{J}}(z, J)<r\right\}
$$

also forms angles $\theta=\theta(r)$ with $\mathbb{R}$. We choose the notation $G_{\theta}(J)$ for this neighborhood. The Schwarz Lemma implies that $G_{\theta}(J) \subset D_{\theta}(J)$.

For the rest of this section we adopt the following notation:

$$
\begin{gathered}
I_{m}=\varphi\left(\left[1, B^{q_{m}}(1)\right]\right), \quad T_{m}=\varphi\left(\left[1, B^{q_{m}-q_{m+1}}(1)\right]\right), \\
G_{m}=G_{m, \alpha}=G_{\alpha}\left(\varphi\left(\left[B^{q_{m+1}}(1), B^{q_{m}-q_{m+1}}(1)\right]\right)\right),
\end{gathered}
$$

where in the definition of the hyperbolic neighborhood $G_{m}$ we fix an angle $0<\alpha<\pi / 2$ which will be specified below. Note that $I_{m} \subset T_{m} \subset$ $\varphi\left(\left[B^{q_{m+1}}(1), B^{q_{m}-q_{m+1}}(1)\right]\right)$ and, by real a priori bounds, the three intervals have 
commensurable lengths. Before giving a result by Yampolsky, let us make the following selections.

- The lifted puzzle pieces $\hat{P}_{n}$. We denote by $\hat{P}_{n}$ the preimage under $\psi$ of $P_{n}$.

- The integer $N$. By Remark 6.1 we may choose some $N \geq 1$ such that $\hat{P}_{n}$ is bounded.

- The angle $\alpha$. We choose $0<\alpha<\pi / 2$ such that

$$
\hat{P}_{N+2} \cup \hat{P}_{N+3} \subset G_{\alpha}\left(\varphi\left(\left[B^{\circ q_{N+2}}(1), B^{q_{N+1}-q_{N+2}}(1)\right]\right)\right)=G_{N+1, \alpha}
$$

and we set $G_{n}=G_{n, \alpha}$ as in (6).

Note that by Corollary 6.1, $P_{n+2} \subseteq P_{n}$ for all $n$, hence $\hat{P}_{n} \subset G_{N+1}$ for all $n \geq N+2$. Since $B$ is the composition of a cubic map with a Möbius transformation, it belongs to an Epstein class Y Ya. Then, the Main Lemma in [Ya can be written in our notation as follows.

Proposition 6.4 (Yampolsky). Let $P_{n}$ denote the nth critical puzzle piece and let $N$ be as above. Then, there exist constants $K_{1}, K_{2}>1$ such that for every $n \geq N+3$ and every $z \in \hat{P}_{n-1}$ with the corresponding backward orbit $\left\{z_{-i}\right\}$ as in (5),

$$
\frac{\operatorname{dist}\left(z_{-\left(q_{n}-1\right)}, J_{-\left(q_{n}-1\right)}\right)}{\left|J_{-\left(q_{n}-1\right)}\right|} \leq K_{1} \frac{\operatorname{dist}\left(z, J_{0}\right)}{\left|J_{0}\right|}+K_{2} .
$$

As a consequence, there exist positive constants $A_{1}, A_{2}$ such that for all $n \geq N+3$,

$$
\frac{\operatorname{diam} P_{n}}{\left|\left[1, B^{-q_{n}}(1)\right]\right|} \leq A_{1} \sqrt[3]{\frac{\operatorname{diam} P_{n-1}}{\left|\left[B^{-q_{n-1}}(1), 1\right]\right|}}+A_{2} .
$$

In the proof of the Proposition 6.4 Yampolsky makes use of the following remark ([Ya, Lemma 4.4):

\section{Remark 6.2. Either}

(1) there exists a moment $i$ of the form $i=j q_{l+1}$ for some $1 \leq j \leq a_{l+2}+1$ and $m \leq l \leq n-2$ such that $\left(\widehat{z_{-i}, J_{-i}}\right)>\epsilon^{*}$ and $\operatorname{dist}\left(z_{-i}, J_{-i}\right)<C^{*}\left|I_{l}\right|$, or

(2) $z_{-q_{n}} \in G_{S}\left(\left[\varphi\left(B^{\left(q_{n-1}-q_{n-2}\right)}(1)\right), 0\right]\right)$ for some $S$ independent of $n$, where $\epsilon^{*}$ and $C^{*}$ depend on the Epstein class of $B$.

The estimate (8) implies that if $\frac{\operatorname{diam} P_{n-1}}{\left|\left[B^{-q_{n-1}}(1), 1\right]\right|}>K$ for a large $K>0$, then

$$
1 \leq \frac{\operatorname{diam} P_{n}}{\left|\left[B^{-q_{n}}(1), 1\right]\right|} \leq \frac{1}{2} \cdot \frac{\operatorname{diam} P_{n-1}}{\left|\left[B^{-q_{n-1}}(1), 1\right]\right|} .
$$

It follows that for large $n$ the puzzle piece $P_{n}$ is commensurable with its base arc $\left[B^{-q_{n}}(1), 1\right]$. By Remark 6.2 combined with the Schwarz Lemma, there exists a constant $\rho>0$ independent of $n$ such that $\hat{P}_{n} \subset G_{\sigma}\left(\left[\varphi\left(B^{\left(q_{n-1}-q_{n-2}\right)}(1)\right), 0\right]\right)$. By the combinatorics of the closest returns, the number of times the pullback of

$$
G_{\sigma}\left(\left[0, \varphi\left(B^{\left(q_{n-2}-q_{n-3}\right)}(1)\right)\right]\right) \supset \hat{P}_{n-1}
$$

along the backward orbit of $\hat{P}_{n}$,

$$
\Pi_{0}=\hat{P}_{n-1}, \Pi_{-1}, \ldots, \Pi_{-q_{n}}=\hat{P}_{n},
$$

hits 0 is bounded by a constant independent of $n$. By the Schwarz Lemma and the elementary properties of the cube root map we have the following. 
Corollary 6.2. There exists an angle $0<\gamma<\pi / 2$ such that for all $n$,

$$
\hat{P}_{n} \subset G_{\gamma}\left(\varphi\left(\left[B^{-q_{n}}(1), 1\right]\right)\right) .
$$

Moreover, by Proposition 6.4. the sequence $\left\{P_{n}\right\}$ induces a fundamental system of connected neighborhoods of 1 in $J\left(\tilde{B}_{\gamma}\right)$ and we have:

Corollary 6.3. If $\gamma$ is an irrational number, then $J\left(\tilde{B}_{\gamma}\right)$ is locally connected at 1 .

By Propositions 6.4 and 6.2, and Remark 2.3, it follows that:

Corollary 6.4 (Only two drop-chains). There are exactly two drop-chains of the form $D_{1}=\overline{\bigcup_{k} U_{i_{1} \ldots i_{k}}}$ and $D_{2}=\overline{\bigcup_{k} U_{i_{1}^{\prime} \ldots i_{k}^{\prime}}}$ accumulating at the critical point 1 . Moreover, both of these drop-chains land at 1 , and they separate $U_{1}$ from $\mathbb{D}$, in the sense that $U_{1}$ and $\mathbb{D}$ belong to different components of $\widehat{\mathbb{C}} \backslash\left(D_{1} \cup D_{2}\right)$.

Thus, directly, we have the following result.

Corollary 6.5. If $\gamma$ is an irrational number of bounded type, then $J\left(\tilde{B}_{\gamma}\right)$ and $J\left(F_{\gamma}\right)$ are locally connected.

Proof (Theorem 6.1). The first step is to prove it for limbs of generation 1.

Lemma 6.2. Let $L_{i}^{\prime}$ be the limb of generation 1 with root $x_{i} \in \mathbb{T}$. Then $\operatorname{diam} L_{i}^{\prime} \rightarrow 0$ as $i$ goes to infinity.

Proof. Let $P_{n}$ be the $n$th critical puzzle piece. We have that

$$
\mathbb{T} \subset \bigcup_{j=0}^{q_{n}-1} B_{\gamma}^{\circ q_{n}-j}\left(P_{n}\right) \cup \bigcup_{j=0}^{q_{n-1}-1} B_{\gamma}^{\circ q_{n+1}-j}\left(P_{n+1}\right)
$$

by the dynamical partition of level $n$ for the homeomorphism $\left(\left.B_{\gamma}\right|_{\mathbb{T}}\right)^{-1}$.

By Corollary 6.2 and the Schwarz Lemma, each piece in the above union has diameter commensurable to its base arc, which uniformly tends to 0 as $n$ goes to infinity, by real a priori bounds. By Proposition6.2, every limb $L_{i}^{\prime}$ with $i \geq q_{n-1}+q_{n}$ is contained in the above union, then $\operatorname{diam} L_{i}^{\prime} \rightarrow 0$ as $i$ goes to infinity.

To finish the proof, observe that for limbs of generation greater than 1, we have two cases. In the first case, the root of the limb is in the boundary of some drop $U_{i_{1} \cdots i_{k}}$ contained in some $\operatorname{limb} L_{i_{1}}^{\prime}$, with $i_{1} \neq 1$, hence we are in the case of the previous lemma. For the other case, when the limb has its root in a drop $U_{1 i_{2} \cdots i_{k}}$, applying the Koebe Distortion Theorem, we have the result which completes the proof.

Corollary 6.6. If $\gamma \in \mathbb{T}$ is an irrational number, then

$$
\lim _{k \rightarrow \infty} \overline{L_{i_{1} i_{2} \ldots i_{k}}^{\prime}}=p .
$$

\section{The PRoOF OF THE MAIN THEOREM}

Let $\gamma \in[0,1 / 2]$ be an irrational number of bounded type and let $c \in \partial W_{0}$ be a parameter with internal argument $\gamma$. Since $P_{c}$ is quasiconformally conjugate to $\tilde{Q}_{\gamma}$ in the corresponding Julia sets and $F_{\gamma}$ is quasiconformally conjugate to $\tilde{B}_{\gamma}$ in the corresponding Julia sets, to prove the Main Theorem it is enough to construct a semi-conjugacy between $\tilde{Q}_{\gamma}$ and $\tilde{B}_{\gamma}$ in the corresponding filled Julia sets. Also, we need to show that the Julia set of $P_{c^{\prime}}$ lives in $J\left(\tilde{B}_{\gamma}\right)$ and that satisfies the condition of the mating. 
7.1. Semi-conjugacy between the models. To show that it is possible to see the filled Julia set $K\left(P_{c}\right)$ in the Fatou set of the quadratic rational map $F_{\gamma}$, it is enough to prove the following result.

Theorem 7.1. Let $\gamma \in[0,1 / 2]$ be an irrational number of bounded type. Then, there exists a continuous map $\varphi_{\gamma}$ from $K\left(\tilde{Q}_{\gamma}\right)$ onto $\widehat{\mathbb{C}}$ such that

$$
\varphi_{\gamma} \circ \tilde{Q}_{\gamma}=\tilde{B}_{\gamma} \circ \varphi_{\gamma}
$$

and it can be chosen quasiconformal in the interior of $K\left(\tilde{Q}_{\gamma}\right)$.

Proof. To define the map on $\mathbb{D}$, we look at the following commutative diagram:

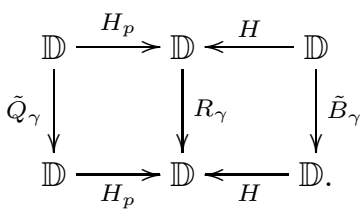

Since $H$ and $H_{p}$ are two quasiconformal homeomorphisms and they can be extended to the boundary of the unit disk, we can define $\varphi_{\gamma}(z)=H^{-1} \circ H_{p}(z)$, for every $z \in \overline{\mathbb{D}}$. If $z$ belongs to a drop $U_{i_{1} \ldots i_{k}}$ of order $m$, that is, $z \in \tilde{Q}_{\gamma}^{-m}(\mathbb{D})$, then $\varphi_{\gamma}(z)$ is the only point inside the drop $U_{i_{1} \ldots i_{k}}^{\prime}$ of $\tilde{B}_{\gamma}^{-m}(\mathbb{D})$, that satisfies

$$
\varphi_{\gamma}(z)=\left.\tilde{B}_{\gamma}^{-m} \circ \varphi_{\gamma}\right|_{\mathbb{D}} \circ \tilde{Q}_{\gamma}^{\circ m}(z) .
$$

Since $\varphi_{\gamma}$ is defined in the closure of the unit disk, using (9), we can extend $\varphi_{\gamma}$ to the closure of any drop. By Proposition 3.3, a point $x \in K\left(\tilde{Q}_{\gamma}\right)$ is either in the closure of a drop or is the limit point of a drop-chain $\mathcal{C}$. Since $\varphi_{\gamma}$ is already defined on $\bigcup_{k} \bigcup_{i_{1} \cdots i_{k}} \bar{U}_{i_{1} \cdots i_{k}}$, it suffices to define it at the landing points of drop-chains of $\tilde{Q}_{\gamma}$.

Let $\mathcal{C}=\overline{\bigcup_{k} U_{i_{1} \cdots i_{k}}}$ be a drop-chain of $\tilde{Q}_{\gamma}$ which lands at $p$, and consider the corresponding drop-chain of $\tilde{B}_{\gamma}, \mathcal{C}^{\prime}=\overline{\bigcup_{k} U_{i_{1} \cdots i_{k}}^{\prime}}$ whose drops have the same addresses. By Theorem 6.1 the diameters of the corresponding limbs $L_{i_{1} \cdots i_{k}}^{\prime}$ go to zero as $k$ goes to infinity, hence $\mathcal{C}^{\prime}$ lands at a well-defined point $p^{\prime} \in K\left(\tilde{B}_{\gamma}\right)$. We define $\varphi_{\gamma}(p)=p^{\prime}$. By definition $\varphi_{\gamma}$ sends any limb $L_{i_{1} \cdots i_{k}}$ of $\tilde{Q}_{\gamma}$ onto the limb $L_{i_{1} \cdots i_{k}}^{\prime}$ of $\tilde{B}_{\gamma}$ with the same address. Next, we will show that $\varphi_{\gamma}$ is a continuous map from $K\left(\tilde{Q}_{\gamma}\right)$ onto $\hat{\mathbb{C}}$.

Let $p \in K\left(\tilde{Q}_{\gamma}\right)$ and let $\left\{p_{n}\right\}$ be a sequence converging to $p$. If $p$ belongs to the interior of $K\left(\tilde{Q}_{\gamma}\right)$, then $\varphi_{\gamma}$ is continuous at $p$. Then, we can assume that $p$ is in the boundary of $K\left(\tilde{Q}_{\gamma}\right)$. By Proposition 3.3, we have two cases:

- Case 1. The point $p$ is the landing point of a drop-chain $\mathcal{C}=\overline{\bigcap_{k} U_{i_{1} \cdots i_{k}}}$. Let us fix a multi-index $i_{1} \cdots i_{k}$ and observe that $p$ belongs to the wake $W_{i_{1} \cdots i_{k}}$. In particular, $p_{n} \in L_{i_{1} \cdots i_{k}}$ which implies $\varphi_{\gamma}\left(p_{n}\right) \in L_{i_{1} \cdots i_{k}}^{\prime}$. Thus, $\operatorname{dist}\left(\varphi_{\gamma}(p), \varphi_{\gamma}\left(p_{n}\right)\right) \leq \operatorname{diam}\left(L_{i_{1} \cdots i_{k}}^{\prime}\right)$. By Theorem 6.1, $\operatorname{diam}\left(L_{i_{1} \cdots i_{k}}^{\prime}\right) \rightarrow 0$ as $k$ goes to infinity, therefore $\varphi_{\gamma}\left(p_{n}\right)$ converge to $\varphi_{\gamma}(p)$ as $n$ goes to infinity.

- Case 2. The point $p$ is in the boundary of a drop $U_{i_{1} \cdots i_{k}}$ of $\tilde{Q}_{\gamma}$ of smallest possible generation. It might be the case that $p$ is the root of a child $U_{i_{1} \cdots i_{k} i_{k+1}}$, in that case $\partial U_{i_{1} \cdots i_{k}} \cap \partial U_{i_{1} \cdots i_{k} i_{k+1}}=\{p\}$. If for all sufficiently large $n, p_{n}$ belongs to $\bar{U}_{i_{1} \cdots i_{k}}$ or $p_{n}$ belongs to $\bar{U}_{i_{1} \cdots i_{k}} \cup \bar{U}_{i_{1} \cdots i_{k} i_{k+1}}$, then we have that $\varphi_{\gamma}\left(p_{n}\right)$ converges to $\varphi_{\gamma}(p)$. 
Therefore, the case when $p_{n} \notin \bar{U}_{i_{1} \cdots i_{k}}$ (or $p_{n} \notin \bar{U}_{i_{1} \cdots i_{k}} \cup \bar{U}_{i_{1} \cdots i_{k} i_{k+1}}$ if $p$ is the root of $\left.U_{i_{1} \cdots i_{k} i_{k+1}}\right)$ remains. Since $p_{n} \rightarrow p$, there exists a limb $L(n)$ with root $x_{n} \in \partial U_{i_{1} \cdots i_{k}}$ such that $x_{n} \rightarrow p$ as $n$ goes to infinity. Hence $\varphi_{\gamma}\left(p_{n}\right)$ belongs to a limb $L^{\prime}(n)$ of $\tilde{B}_{\gamma}$ with the same address as $L(n)$ and whose root $x_{n}^{\prime}=\varphi_{\gamma}\left(x_{n}\right)$ converges to $\varphi_{\gamma}(p)$ as $n$ goes to infinity. By Theorem 6.1 $\operatorname{diam}\left(L^{\prime}(n)\right) \rightarrow 0$, then $\varphi_{\gamma}\left(p_{n}\right) \rightarrow \varphi_{\gamma}(p)$ as $n$ goes to infinity.

By definition $\varphi_{\gamma}(z)$ is quasiconformal in the interior of $K\left(\tilde{Q}_{\gamma}\right)$ and it is surjective by Lemma 4.2 .

Since $\varphi_{\gamma}\left(K\left(\tilde{Q}_{\gamma}\right)\right)=\widehat{\mathbb{C}}$, to finish the proof of the Main Theorem it is enough to show that the Julia set of $P_{c^{\prime}}$ also lives in $J\left(F_{\gamma}\right)$ and satisfies the condition of gluing in the mating. We have chosen $c^{\prime} \in \mathbb{R}$ such that the critical value $c^{\prime} \in J_{c^{\prime}}$ of $P_{c^{\prime}}$ has external arguments $\frac{1}{2}-\frac{\theta}{4}$ and $\frac{1}{2}+\frac{\theta}{4}$, where $\theta$ is the external argument of $c \in J_{c}$.

If $R_{c}(t)$ and $R_{c^{\prime}}(t)$ denote the external rays of angles $t \in \mathbb{T}$ for $K_{c}$ and $K_{c^{\prime}}$, respectively, then in the mating $R_{c}(t) \sim R_{c^{\prime}}(-t)$. In particular, $c$ and $P_{c^{\prime}}^{\circ 2}\left(c^{\prime}\right)$ are equivalent and

$$
P_{c^{\prime}}^{\circ 2+k}\left(c^{\prime}\right) \sim P_{c}^{\circ k}(c), \quad k \in \mathbb{N},
$$

which is a dense set in the boundary of the Siegel disk of $K_{c}$.

Since we have defined $\varphi_{\gamma}$ in $K\left(\tilde{Q}_{\gamma}\right)$ and $\tilde{Q}_{\gamma}$ is quasiconformally equivalent to $P_{c}$, we have a quasiconformal homeomorphism $\Phi=\varphi_{\gamma} \circ \psi_{\gamma}$ that conjugates $P_{c}$ to $\tilde{Q}_{\gamma}$ in $K_{c}$; therefore, we can extend $\Phi$ to $X=\left(K_{c^{\prime}} \sqcup K_{c}\right) / \sim$.

To see $J_{c^{\prime}}$ in $J\left(\tilde{Q}_{\gamma}\right)$, we send 0 in -1 under $\Phi$. Thus $\Phi\left(P_{c^{\prime}}^{\circ k}(0)\right)=\tilde{B}_{\gamma}^{\circ k}(-1)=$ $\tilde{B}_{\gamma}^{\circ k-2}(1)$, which is the condition required in the mating. Hence $\Phi$ is well defined in the postcritical orbit and it is continuous because $P_{c^{\prime}}$, restricted to the postcritical orbit, is semi-conjugate to the rigid rotation of angle $\gamma$.

To define $\Phi$ in all $J_{c^{\prime}}$, first of all, we will define it at all the preimages of the critical point -1 which is a dense subset of $J\left(\tilde{B}_{\gamma}\right)$.

In $K\left(\tilde{Q}_{\gamma}\right)$, we consider the two drop-chains

$$
\mathcal{C}=\overline{U_{0} \cup U_{1} \cup U_{11} \cup \cdots}, \quad \mathcal{C}^{\prime}=\overline{U_{0} \cup U_{2} \cup U_{21} \cup \cdots}
$$

with $\tilde{Q}_{\gamma}\left(\mathcal{C}^{\prime}\right)=\mathcal{C}$.

By construction, $\mathcal{C}^{\prime}$ and $\mathcal{C}$ land at the repelling fixed point $\beta_{c}$ and its preimage $\beta_{c}^{\prime}$, respectively YaZa. We define the spine of $\tilde{Q}_{\gamma}$ as the union of the drop-rays

$$
S_{\gamma}=R(\mathcal{C}) \cup R\left(\mathcal{C}^{\prime}\right),
$$

and $S_{\gamma}^{\prime}=\psi_{\gamma}\left(S_{\gamma}\right) \subset K_{c}$. For $c^{\prime} \in \mathbb{R}$, the spine for $K_{c^{\prime}}$ is the interval $\left[-\beta_{c^{\prime}}, \beta_{c^{\prime}}\right]$.

Taking the union of $R_{c}(0), R_{c}\left(\frac{1}{2}\right)$ and $S_{\gamma}^{\prime}$, we get a curve that decomposes the dynamical plane of $P_{c}$ in two components; this decomposition allows us to write the external argument of any $z \in J_{c}$ in binary expansion YaZa. We call the upper component the one that contains the points $z \in J_{c}$ with external argument in the interval $\left(0, \frac{1}{2}\right)$. In a similar way, we have that the union of $R_{c^{\prime}}(0), R_{c^{\prime}}\left(\frac{1}{2}\right)$ and the spine of $K_{c^{\prime}}$ is the whole real line, and the upper component is the upper-half plane.

Since $c$ and $P_{c^{\prime}}^{\circ 2}\left(c^{\prime}\right)$ belong to the same class, using the above decomposition, we can choose a preimage of $c$ and $c^{\prime}$, under $P_{c}$ and $P_{c^{\prime}}$, respectively, in one of the two components and they are mapped by $\Phi$ to the same point. Then we have that in the topological mating $P_{c^{\prime}}^{\circ 2-k}\left(c^{\prime}\right)$ is equivalent to $P_{c}^{\circ-k}(c)$. Note that $K\left(\tilde{B}_{\gamma}\right)$ has a Siegel disk that contains two critical points -1 and 1 in the boundary. Since the 
map $\Phi$ sends $c$ to 1 , and $\tilde{B}_{\gamma}^{\circ 2}(-1)=1$, we have that $\tilde{B}_{\gamma}$ satisfies the condition of the mating in $c$.

Since $\left\{P_{c}^{\circ-k}(c)\right\}$ is a dense set in $J_{c}$, it follows that the condition of the mating between $K_{c}$ and $K_{c^{\prime}}$ is satisfied in a dense set of $J\left(\tilde{B}_{\gamma}\right)$. By the continuity of $\varphi_{\gamma}$ in $K\left(\tilde{Q}_{\gamma}\right)$, we have the mating condition. In order to finish, taking $\Theta(z)=\varsigma_{\gamma} \circ \Phi$, we have the conjugation on $K_{c} \sqcup K_{c^{\prime}} / \sim$. Since $\Theta=\varsigma_{\gamma} \circ \varphi_{\gamma} \circ \psi_{\gamma}$, this conjugation is conformal in the interior of $K_{c}$.

\section{ACKNOWLEDGMENTS}

The first author would like to thank Adrien Douady for suggesting this problem. We would like to thank Carsten Petersen for his valuable comments about local connectivity of Julia sets. Also, we are indebted to Michael Yampolsky for his valuable comments about critical circle maps. We express our gratitude to IMUNAM (Unidad Cuernavaca) for their hospitality during the elaboration of the last part of this paper.

\section{REFERENCES}

[Ah] L.V. Ahlfors, Lectures on quasiconformal mappings, Van Nostrand Math. Studies, No. 10, D. Van Nostrand Co. Inc., New York, 1966. MR0200442 (34:336)

[B] A.F. Beardon, Iteration of rational functions, Springer-Verlag, 1991. MR.1128089 (92j:30026)

[Bl] G. Ble, External arguments and invariant measures for the quadratic family, Disc. and Cont. Dyn. Sys. 11 (2004), 241-260. MR2083418 (2005e:37097)

[CG] L. Carleson and T.W. Gamelin, Complex Dynamics, Springer-Verlag, 1993. MR.1230383 (94h:30033)

[CL] E. F. Collingwood and A.J. Lohwater, The theory of cluster sets, Cambridge at the University Press, 1966. MR0231999 (38:325)

[D] A. Douady, Systèmes Dynamiques Holomorphes, Séminaire Bourbaki, 35é année \# 599, Astérisque 105-106 (1983), 39-63. MR0728980 (85h:58090)

[D2] A. Douady, Disques de Siegel et anneaux de Herman, Séminaire Bourbaki, 1986-87, exposé No.677, Astérisque 152-153, (1987), 151-172. MR0936853(89g:30049)

[DE] A. Douady and C.J. Earle, Conformally natural extension of homeomorphismes of the circle, Act. Math. 157 (1986), 25-48. MR0857678 (87j:30041)

[DH] A. Douady and J.H. Hubbard, Étude dynamique des polynômes complexes I et II, Pub. Math. d'Orsay 84-02 and 85-02, (1984-85).

[Ep] A. Epstein, Counterexamples to the quadratic mating conjecture, Manuscript 1998.

[F] P. Fatou, Mémoire sur les équations fonctionnelles, Bull. S.M.F 47 (1919), 161-271; 48 (1920), 33-94 and 208-314.

[HW] G.H. Hardy and E.M. Wright, An introduction to the theory of numbers, Oxford University Press, 1979. MR0568909 (81i:10002)

[LV] O. Lehto and J. Virtanen, Quasiconformal Mappings in the Plane, Springer-Verlag, 1973. MR.0344463 (49:9202)

[dMvS] W. de Melo and S. van Strien, One-dimensional dynamics, Springer Verlag, 1993. MR 1239171 (95a:58035)

[M1] J. Milnor, Pasting together Julia sets: A worked out example of mating, Experimen. Math. 13 (2004), 55-92. MR2065568 (2005c:37087)

[M2] J. Milnor, Geometry and dynamics of quadratic rational maps, Experimen. Math. 2 (1993), 37-83. MR1246482(96b:58094)

[Pe] C.L. Petersen, Local connectivity of some Julia sets containing a circle with an irrational rotation, Act. Math. 177 (1996), 163-224. MR1440932 (98h:58164)

[Pe1] C.L. Petersen, The Herman-Światek theorem, with applications, The Mandelbrot Set, theme and variations. London Mathematical Society, Lecture Note Series 274 (2000), 211-225. MR1765090 (2001b:37061) 
[PeZa] C.L. Petersen and S. Zakeri, On the Julia set of a typical quadratic polynomial with a Siegel disk, Ann. of Math. (2) 159 (2004), 1-52. MR2051390 (2005c:37085)

[Re] M. Rees, Realization of matings of polynomials as rational maps of degree two, Manuscript 1986.

[Sh] M. Shishikura, On a theorem of M. Rees for matings of polynomials, Preprint IHES, 1990.

[S] D. Sullivan, Quasiconformal homeomorphisms and dynamics I: Solution of the FatouJulia problem on wandering domains, Ann. of Math. 122 (1985), 401-418. MR0819553 (87i:58103)

[TL] T. Lei, Accouplements de polynômes complexes, Ph.D. Thesis, Université Paris-Sud 1987.

[Ya] M. Yampolsky, Complex bounds for renormalization of critical circle maps, Erg. Th. and Dyn. Sys. 19 (1999), 227-257. MR.1677153 (2000d:37053)

[YaZa] M. Yampolsky and S. Zakeri, Mating Siegel quadratic polynomials, J. Am. Math. Soc. 14, No. 1, (2001), 25-78. MR1800348 (2001k:37064)

[Y] J.C. Yoccoz, Il n'y a pas de contre-example de Denjoy analytique, C. R. Acad. Sci. Paris 298 (1984), 141-144. MR0741080 (85j:58134)

División Académica de Ciencias Básicas, Universidad Juárez Autónoma de Tabasco, Km. 1 Carr. Cunduacán-Jalpa, C.P. 86690, Cunduacán, Tabasco, México

E-mail address: gble@ujat.mx

Facultad de Ciencias, Universidad Autónoma del Estado de Morelos, Av. Universidad 1001, col. Lomas de Chamilpa, C.P. 62210 Cuernavaca, Morelos, México

E-mail address: rogelio@matcuer.unam.mx 